\title{
Encrypted DNS $\Longrightarrow$ Privacy? A Traffic Analysis Perspective
}

\author{
Sandra Siby*II , Marc Juarez ${ }^{\dagger}$ II, Claudia Diaz ${ }^{\ddagger}$, Narseo Vallina-Rodriguez ${ }^{\S}$ and Carmela Troncoso* \\ * EPFL, \{sandra.siby, carmela.troncoso\}@epfl.ch \\ $\dagger$ University of Southern California, marc.juarez@usc.edu \\ $\ddagger$ imec-COSIC KU Leuven, claudia.diaz@esat.kuleuven.be \\ $\S$ IMDEA Networks Institute, narseo.vallina@imdea.org
}

\begin{abstract}
Virtually every connection to an Internet service is preceded by a DNS lookup. Lookups are performed without any traffic-level protection, thus enabling manipulation, redirection, surveillance, and censorship. To address these issues, large organizations such as Google and Cloudflare are deploying standardized protocols that encrypt DNS traffic between end users and recursive resolvers: DNS-over-TLS (DoT) and DNS-over-HTTPS (DoH). In this paper, we examine whether encrypting DNS traffic can protect users from traffic analysis-based monitoring and censoring. We propose a novel feature set to perform traffic analysis attacks, as the features used to attack HTTPS or Tor traffic are not suitable for DNS' characteristics. We show that traffic analysis enables the identification of domains with high accuracy in closed and open world settings, using 124 times less data than attacks on HTTPS flows. We also show that DNS-based censorship is still possible on encrypted DNS traffic. We find that factors such as end-user location, recursive resolver, platform, or DNS client do negatively affect the attacks' performance, but they are far from completely stopping them. We demonstrate that the standardized padding schemes are not effective. Yet, Tor - which does not effectively mitigate traffic analysis attacks on web traffic- is a good defense against DoH traffic analysis.
\end{abstract}

\section{INTRODUCTION}

Regular Domain Name System (DNS) requests have been mostly sent in the clear [1]. This situation enables entities, such as Internet Service Providers (ISPs), Autonomous Systems (ASes), or state-level agencies, to perform user tracking, mass surveillance $[2,3]$ and censorship $[4,5]$. The risk of pervasive surveillance and its consequences has prompted Internet governance, industry actors, and standardization bodies to foster privacy protections [6, 7]. In particular, for DNS these bodies have standardized two protocols: DNS-over-TLS (DoT) [8] and DNS-over-HTTPS (DoH) [9]. These protocols encrypt the communication between the client and the recursive resolver to prevent the inspection of domain names by network eavesdroppers. These standarization bodies also consider protection mechanisms to limit inference of private information from traffic metadata, such as the timing and size

\footnotetext{
पI The authors contributed equally to this paper. M. Juarez did most of this research while at $\mathrm{KU}$ Leuven.
}

Network and Distributed Systems Security (NDSS) Symposium 2020 23-26 February 2020, San Diego, CA, USA

ISBN 1-891562-61-4

https://dx.doi.org/10.14722/ndss.2020.24301

www.ndss-symposium.org of network packets of the encrypted DNS communication. These mechanisms protect against traffic analysis by padding traffic [10], or by multiplexing the encrypted DNS traffic with other traffic, e.g., when DoH and web HTTPS traffic share a single TLS tunnel (see $\S 8.1$ [9]).

During 2018, Google and Cloudflare launched public DoH resolvers [11, 12], while Mozilla added DoH support to Firefox [13] and has been gradually rolling it out as the default configuration for Firefox users in the US since September 2019 [14]. These efforts aim to leverage DoH and DoT's capacity to enhance users' browser traffic security guarantees [15]. Yet, it is known that even when communications are encrypted, traffic features such as volume and timing can reveal information about their content [16, 17, 18, 19, 20,21]. As of today, existing evaluations of DoH implementations have focused on understanding the impact of encryption and transport protocols on performance $[22,23]$, and on cost $[24,25]$.

In this paper, we aim to fill this gap by studying the effectiveness of traffic analysis attacks in revealing users' browsing patterns from encrypted DNS. We focus our analysis on $\mathrm{DoH}$, as its adoption by large industry actors (e.g., Google, Cloudflare, and Mozilla) makes it prevalent in the wild. For completeness, we include a comparison of the protection provided by Cloudflare's DoH and DoT resolvers. In our analysis, we consider a passive adversary, as described in RFC 7626 [6], who is placed between the client and the DNS resolver. The adversary's goal is to identify which web pages users visit, to either perform surveillance or censorship. As the RFC stresses, this adversary may be in "a different path than the communication between the initiator [e.g., the client] and the recipient [e.g., a web server]" [6], and thus can launch attacks even if they do not see all the traffic between endpoints.

We find that features traditionally used in atttacks on web traffic [16, 17, 18, 19, 26, 27] are not suitable for encrypted DNS traffic. This is because DNS, as opposed to web traffic, is bursty, chatty, and is mostly composed of small packets. We engineer a novel set of features that focuses on local traffic features, and enables successful identification of requested websites on encrypted DNS. As encrypted DNS traces are much smaller that their web traffic counterparts, our techniques require 124 times less data than state-of-the-art traffic analysis on web traffic, allowing adversaries to run attacks at large scale [28]. Furthermore, our new feature set on encrypted DNS 
traffic is as effective or more so than state-of-the-art attacks on web traffic in identifying web pages.

We also find that differences between the environment used by the adversary to train the attack (e.g., user location, choice of client application, platform or recursive DNS resolver), and the environment where the attack is actually deployed, negatively affect the performance of the attack. Most prior work on traffic analysis assumes the adversary knows the environment where the attack will be deployed, but the adversary cannot trivially obtain that information a priori. Our features allow the adversary to infer that information and thus tailor the attacks accordingly, maintaining high attack performance for each specific environment.

Next, we evaluate existing traffic analysis defenses, including the standardized EDNSO padding [10] —implemented by Cloudflare and Google in their solutions-, and the use of Tor [29] as transport, a feature available when using Cloudflare's resolver. We find that, unless EDNS0 padding overhead is large, current padding strategies cannot completely prevent our attack. Also, while Tor offers little protection against web page fingerprinting on web traffic $[17,18,19,30]$, Tor is an extremely effective defense against web page fingerprinting on encrypted DNS traffic.

Finally, we measure the potential of encryption to hinder DNSbased censorship practices. We show that with encryption, it is still possible to identify which packet carries the DNS lookup for the first domain. We quantify the collateral damage of blocking the response to this lookup, thereby preventing the user from seeing any content. We also show that, to minimize the effect of censorship on non-blacklisted pages, censors must wait to see, on average, $15 \%$ of the encrypted DNS traffic.

Our main contributions are as follows:

- We show that the features for traffic analysis existing in the literature are not effective on encrypted DNS. We propose a new feature set that results in successful attacks on encrypted DNS and that outperforms existing attacks on HTTPS (Section V-A).

- We show that web page fingerprinting on DoH achieves the same accuracy as web page fingerprinting on encrypted web traffic, while requiring 124 times less volume of data. We show that factors such as end-user location, choice of DNS resolver, and client-side application or platform, have a negative impact on the effectiveness of the attacks, but do not prevent them (Section V).

- We evaluate the traffic analysis defenses proposed in the standard and show that they are not effective. We find that in the case of encrypted DNS, contrary to web traffic, routing over Tor deters web page identification on encrypted DNS traffic (Section VI).

- We evaluate the feasibility of DNS-based censorship when DNS lookups are encrypted. We show the censor can identify the packet with the first domain lookup. We quantify the tradeoff between how much content from a blacklisted page the user can download, and how many non-blacklisted pages are censored as a side effect of traffic-analysis-based blocking (Section VII).

- We gather the first dataset of encrypted DNS traffic collected in a wide range of environments (Section IV). ${ }^{1}$

Impact Upon responsible disclosure of our attacks, Cloudflare changed their DoH resolver to include padding. This work was also invited to an IETF Privacy Enhancements and Assessments Research Group Meeting, and will contribute to the next RFC for traffic analysis protection of encrypted DNS

\section{BACKGROUND AND RELATED WORK}

In this section, we provide background on the Domain Name System (DNS) and existing work on DNS privacy.

The Domain Name System (DNS) is primarily used for translating easy-to-read domain names to numerical IP addresses $^{2}$. This translation is known as domain resolution. In order to resolve a domain, a client sends a DNS query to a recursive resolver, a server typically provided by the ISP with resolving and caching capabilities. If the domain resolution by a client is not cached by the recursive name server, it contacts a number of authoritative name servers which hold a distributed database of domain names to IP mappings. The recursive resolver traverses the hierarchy of authoritative name servers until it obtains an answer for the query, and sends it back to the client. The client can use the resolved IP address to connect to the destination host. Figure 1 illustrates this process.

Enhancing DNS Privacy. Security was not a major consideration in the first versions of DNS, and for years DNS traffic was sent in the clear over (untrusted) networks. Over the last few years, security and privacy concerns have fostered the appearance of solutions to make DNS traffic resistant to eavesdropping and tampering. Several studies have empirically demonstrated how the open nature of DNS traffic is being abused for performing censorship [33, 34] and surveillance [35, 2]. Early efforts include protocols such as DNSSEC [36] and DNSCrypt [37]. DNSSEC prevents manipulation of DNS data using digital signatures. It does not, however, provide confidentiality. DNSCrypt, an open-source effort, provides both confidentiality and authenticity. However, due to lack of standardization, it has not achieved wide adoption.

In 2016, the IETF approved DNS-over-TLS (DoT) [8] as a Standards Track protocol. The client establishes a TLS session with a recursive resolver (usually on port TCP:853 as standardized by IANA [8]) and exchanges DNS queries and responses over the encrypted connection. To amortize costs, the TLS session between the client and the recursive DNS resolver is usually kept alive and reused for multiple queries. Queries go through this channel in the same manner as in unencrypted DNS - chatty and in small volume.

In DoH, standardized in 2018, the local DNS resolver establishes an HTTPS connection to the recursive resolver and encodes the DNS queries in the body of HTTP requests. DoH considers the use of HTTP/2's Server Push mechanism. This enables the server to preemptively push DNS responses that are likely to follow a DNS lookup [38], thus reducing

\footnotetext{
${ }^{1}$ Dataset and code at: https://github.com/spring-epfl/doh_traffic_analysis

${ }^{2}$ Over time, other applications have been built on top of DNS [31, 32]
} 
communication latency. As opposed to DoT, which uses a dedicated TCP port for DNS traffic and thus is easy to monitor and block, DoH lookups can be sent along non-DNS traffic using existing HTTPS connections (yet potentially blockable at the IP level).

There are several available implementations of DoT and DoH. Since 2018, Cloudflare and Quad9 provide both DoH and DoT resolvers, Google supports DoH, and Android 10 has native support for DoT. DoH enjoys widespread support from browser vendors. Firefox provides the option of directing DNS traffic to a trusted recursive resolver such as a DoH resolver, falling back to plaintext DNS if the resolution over DoH fails. In September 2019, Google announced support for DoH in version 78 of Chrome [39]. Cloudflare also distributes a standalone DoH client and, in 2018, they released a hidden resolver that provides DNS over Tor, not only protecting lookups from eavesdroppers but also providing anonymity for clients towards the resolver. Other protocols, such as DNS-over-DTLS [40], an Experimental RFC proposed by Cisco in 2017, and DNSover-QUIC [41], proposed to the IETF in 2017 by industry actors, are not widely deployed so far.

Several academic works study privacy issues related to encrypted DNS. Shulman suggests that encryption alone may not be sufficient to protect users [42], but does not provide any experiments that validate this statement. Our results confirm her hypothesis that encrypted DNS response size variations can be a distinguishing feature. Herrmann et al. study the potential of DNS traces as identifiers to perform user tracking, but do not consider encryption [43]. Finally, Imana et al. study privacy leaks on traffic between recursive and authoritative resolvers [44]. This is not protected by $\mathrm{DoH}$, and it is out of scope of our study.

\section{PROBlem STATEMENT}

In this paper, we study if it is possible to infer which websites a user visits by observing encrypted DNS traffic. This information is of interest to multiple actors, e.g., entities computing statistics on Internet usage [45, 46], entities looking to identify malicious activities [47, 48, 5], entities performing surveillance $[35,2]$, or entities conducting censorship [49, 34].

We consider an adversary that can collect encrypted DNS traffic between the user and the DNS recursive resolver (red dotted lines in Figure 1), and thus, can link lookups to a specific origin IP address. Such an adversary could be present in the users' local network, near the resolver, or anywhere along the path (e.g., an ISP or compromised network router). As noted in the RFC, this adversary may be "in a different path than the communication between the initiator and the recipient" [6].

This adversary model also includes adversaries that only see DoH traffic, e.g., adversaries located in an AS that lies between the user and the resolver but not between the user and the destination host. In order to confirm that this adversary is possible, we conducted an experiment where we ran traceroutes to a subset of the websites we use in the study (1,445 websites), and to two resolvers - Cloudflare and Google. We intersected the AS sets and observed that the sets do not fully overlap in $93 \%$ and $90 \%$ of the cases for Cloudflare and Google respectively. Furthermore, we note that BGP hijacking attacks, which are becoming increasingly frequent [50], can be used to selectively intercept paths to DoH resolvers. In such cases,

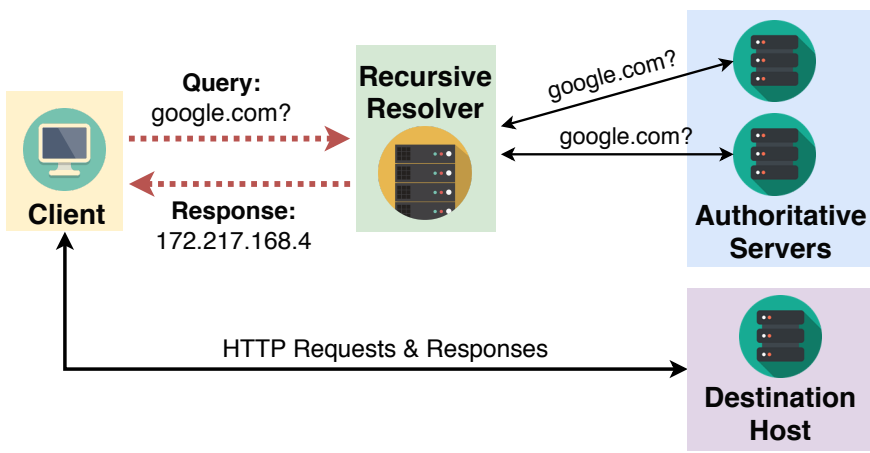

Figure 1: DNS resolution: To visit www . google. com, a user queries the recursive resolver for its IP. If the record is not cached, the recursive resolver queries an authoritative resolver and forwards the response to the client. The client uses the IP in the response to connect to the server via HTTP. We consider an adversary placed between the client and the resolver (i.e., observes the red dotted lines).

the adversary can only rely on DNS fingerprinting to learn which webpages are visited by a concrete user for monitoring, or censorship [2, 35]. If the adversary is actually in the path to the users' destination, she could perform traditional website fingerprinting. However, we show that web page fingerprinting on only DoH traffic achieves the same accuracy while requiring (on average) 124 times less data than attacking HTTPS traces that include web traffic. This is critical to guarantee the scalability of attacks to a large number of targets [28].

We assume that the adversary has access to encrypted DNS traffic traces that are generated when the user visits a website via HTTP/S using DoH to resolve the IPs of the resources. An encrypted DNS trace, which we also call DoH trace, comprises the resolution of the visited website's first-party domain, and the subsequent resolutions for the resources contained in the website, e.g., images and scripts. For instance, for visiting Reddit, after resolving wWw.reddit.com, the client would resolve domains such as cdn.taboola.com, doubleclick.net and thumbs.redditmedia.com, among others.

We consider two adversarial goals. First, monitoring the browsing behavior of users, which we study in Section V; and, second, censoring the web pages that users visit, which we address in Section VII. These two goals differ in their data collection needs. Monitoring adversaries can collect full traces to make their inferences as accurate as possible, as they do not take any action based on their observations. In contrast, censorship adversaries need to find out which domain is being requested as fast as possible so as to interrupt the communication. Thus, they act on partial traces.

\section{Data Collection}

To collect data we set up Ubuntu 16.04 virtual machines with DoH clients that send DNS queries to a public DoH resolver. We use Selenium ${ }^{3}$ (version 3.14.1) to automatically visit a webpage from our list, triggering DNS lookups for its resources. We restart the browser in between webpage visits to ensure that the cache and profile do not affect collection.

\footnotetext{
${ }^{3}$ https://www.seleniumhq.org/
} 
Table I: Overview of datasets. Our default configuration is a Desktop located in Lausanne where Firefox uses Cloudflare's client to query Cloudflare's resolver. Changes are detailed in the table (see Appendix for a detailed version).

\begin{tabular}{ccccc}
\hline Name & Identifier & Location & \# webpages & \# samples \\
\hline Desktop (Location 1) & LOC1 & Lausanne & 1,500 & 200 \\
Desktop (Location 2) & LOC2 & Leuven & 1,500 & 60 \\
Desktop (Location 3) & LOC3 & Singapore & 1,500 & 60 \\
Raspberry Pi & RPI & Lausanne & 700 & 60 \\
Firefox with Google resolver & GOOGLE & Leuven & 700 & 60 \\
Firefox with Cloudflare resolver & CLOUD & Leuven & 700 & 60 \\
Firefox with Cloudflare client & CL-FF & Leuven & 700 & 60 \\
Open World & OW & Lausanne & 5,000 & 3 \\
DoH and web traffic & WEB & Leuven & 700 & 60 \\
DNS over Tor & TOR & Lausanne & 700 & 60 \\
Cloudflare's EDNS0 padding & EDNS0-128 & Lausanne & 700 & 60 \\
Recommended EDNS0 padding & EDNS0-468 & Lausanne & 700 & 60 \\
EDNS0 padding with ad-blocker & EDNS0-128-adblock & Lausanne & 700 & 60 \\
DoT with Stubby client & DOT & Lausanne & 700 & 60 \\
\hline
\end{tabular}

We capture network traffic between the DoH client and the resolver using tcpdump, and we filter the traffic by destination port and IP to obtain the final DoH traffic trace.

We collect traces for the top, middle, and bottom 500 webpages in Alexa's top million websites list on 26 March 2018, 1,500 webpages in total. We note that even though 1,500 is still a small world compared to the size of the Web, the largest world considered in evaluations similar to ours for website fingerprinting on web traffic over Tor is of 800 websites [51, $30,52,18,53,19]$.

We visit each webpage in a round-robin fashion, obtaining up to 200 samples for every webpage. For our open world analysis, we collect traces of an additional 5,000 webpages from the Alexa top million list. We collected data during two periods, from 26 August 2018 to 9 November 2018, and from 20 April 2019 to 14 May 2019. Data from these two periods is never mixed in the analysis.

We consider different scenarios varying DoH client and resolver, end user location and platform, and the use of DNS traffic analysis defenses (padding, ad-blocker usage, DNSover-Tor). Table I provides an overview of the collected datasets. In order to better understand the vulnerability of DNS encryption to traffic analysis, we designed heterogenous experiments that look into different aspects of the problem, resulting in multiple datasets of varied sizes and collected under different conditions - in many cases, several datasets for each experiment type. In each experiment, we vary one characteristic (e.g., location or platform), and keep the default configuration for the rest.

We also collected a dataset (using the Stubby client and Cloudflare's resolver) to study the effectiveness of defenses on DoT traffic as compared to DoH. Since Cloudflare had already implemented padding of responses for DoT traffic at the time of data collection, we were not able to collect a dataset of DoT without padding. In the following sections, we use the Identifier provided in the second column to refer to each of the datasets. Note that unless specified otherwise, we use Cloudflare's DoH client.

Data curation. We curate the datasets to ensure that our results are not biased. First, we aim at removing the effect of spurious errors in collection. We define those as changes in the websites for reasons other than those variations due to their organic evolution that do not represent the expected behavior of the page. For instance, pages that go down during the collection period.

Second, we try to eliminate website behavior that is bound to generate classification errors unrelated to the characteristics of DNS traffic. For instance, different domains generating the same exact DNS traces, e.g., when webpages redirect to other pages or to the same resource; or web servers returning standard errors (e.g., 404 Not Found or 403 Forbidden).

For curation, we use the Chrome over Selenium crawler to collect the HTTP request/responses, not the DNS queries responses, of all the pages in our list in LOC1. We conduct two checks. First, we look at the HTTP response status of the FQDN (Fully Qualified Domain Name), that is being requested by the client. We identify the webpages that do not have an HTTP OK status. These could be caused by a number of factors, such as pages not found (404), anti-bot mechanisms, forbidden responses due to geoblocking [54] (403), internal server errors (500), and so on. We mark these domains as invalid. Second, we confirm that the FQDN is present in the list of requests and responses. This ensures that the page the client is requesting is not redirecting the browser to other URLs. This check triggers some false alarms. For example, a webpage might redirect to a country-specific version (indeed. com redirecting to indeed. fr, results in indeed. com not being present in the list of requests); or in domain redirections (amazonaws. com redirecting to aws. amazon. com). We do not consider these cases as anomalies. Other cases are full redirections. Examples are malware that redirects browser requests to google.com, webpages that redirect to GDPR country restriction notices, or webpages that redirect to domains that specify that the site is closed. We consider these cases as invalid webpages and add them to our list of invalid domains.

We repeat these checks multiple times over our collection period. We find that 70 webpages that had invalid statuses at some point during our crawl, and 16 that showed some fluctuation in their status (from invalid to valid or vice versa). We study the effects of keeping and removing these webpages in Section V-B.

\section{DNS-BASED WEBSITE FINGERPRINTING}

Website fingerprinting attacks enable a local eavesdropper to determine which web pages a user is accessing over an encrypted or anonyimized channel. It exploits the fact that the size, timing, and order of TLS packets are a reflection of a website's content. As resources are unique to each webpage, the traces identify the web even if traffic is encrypted. Website fingerprinting has been shown to be effective on HTTPS [27, $26,55]$, OpenSSH tunnels [56, 57], encrypted web proxies [58, 59] and VPNs [60], and even on anonymous communications systems such as Tor $[61,30,51,52,16,17,18,19]$.

The patterns exploited by website fingerprinting are correlated with patterns in DNS traffic: which resources are loaded, and their order determines the order of the corresponding DNS queries. Thus, we expect that website fingerprinting can also be effective on DNS encrypted flows such as DNS-over-HTTPS $(\mathrm{DoH})$ or DNS-over-TLS (DoT). In this paper, we call DNS fingerprinting the use of traffic analysis to identify the web page that generated an encrypted DNS trace, i.e., website fingerprinting on encrypted DNS traffic. In the following, when- 
ever we do not explicitly specify whether the target of website fingerprinting is DNS or HTTPS traffic, we refer to traditional website fingerprinting on web traffic over HTTPS.

\section{A. DNS fingerprinting}

As in website fingerprinting, we treat DNS fingerprinting as a supervised learning problem: the adversary first collects a training dataset of encrypted DNS traces for a set of pages. The page (label) corresponding to a DNS trace is known. The adversary extracts features from these traces (e.g., lengths of network packets) and trains a classifier that, given a network trace, identifies the visited page. Under deployment, the adversary collects traffic from a victim and feeds it to the classifier to determine which page the user is visiting.

Traffic variability. Environmental conditions introduce variance in the DNS traces sampled for the same website. Thus, the adversary must collect multiple DNS traces in order to obtain a robust representation of the page.

Some of this variability has similar origin to that of web traffic. For instance, changes on the website that result in varying DNS lookups associated with third-party embedded resources (e.g., domains associated to ad networks); the platform where the client runs, the configuration of the DoH client, or the software using the client which may vary the DNS requests (e.g., mobile versions of websites, or browsers' use of pre-fetching); and the effects of content localization and personalization that determine which resources are served to the user.

Additionally, there are some variability factors specific to DNS traffic. For instance, the effect of the local resolver, which depending on the state of the cache may or may not launch requests to the authoritative server; or the DNS-level loadbalancing and replica selection (e.g., CDNs) which may provide different IPs for a given domain or resource [62].

Feature engineering. Besides the extra traffic variability compared to web traffic, DNS responses are generally smaller and more chatty than web resources $[62,63]$. In fact, even when DNS lookups are wrapped in HTTP requests, DNS requests and responses fit in one single TLS record in most cases. These particularities hint that traditional website fingerprinting features, typically based on aggregate metrics of traffic traces such as the total number of packets, total bytes, and their statistics (e.g., average, standard deviation), are inadequate to characterize DoH traffic. We test this hypothesis in the following section, where we show that state-of-the-art web traffic attacks' performance drops in $20 \%$ when applied on DoH traces (see Table III).

To address this problem, we introduce a novel set of features, consisting of n-grams of TLS record lengths in a trace. Following the usual convention in website fingerprinting, we represent a traffic trace as a sequence of integers, where the absolute value is the size of the TLS record and the sign indicates direction: positive for packets from the client to the resolver (outgoing), and negative for the packets from the resolver to the client (incoming). An example of this represenation is the trace: $(-64,88,33,-33)$. Then, the uni-grams for this trace are $(-64),(88),(33),(-33)$, and the bi-grams are $(-64,88),(88,33),(33,-33)$. To create the features, we take tuples of $n$ consecutive TLS record lengths in the DoH traffic traces and count the number of their occurrences in each trace.

In some of our experiments, we used a proxy to man-inthe-middle the DoH connection between the client and the resolver $^{4}$, and obtained the OpenSSL TLS session keys using Lekensteyn's scripts ${ }^{5}$. In all the decrypted TLS records we observe only one single DoH message (either a request or a response). However, as Houser et al. indicate, some clients and resolvers' implementations result on multiple DoT messages in the same TLS record [64].

The intuition behind our choice of features is that n-grams capture patterns in request-response size pairs, as well as the local order of the packet size sequence. To the best of our knowledge, n-grams have never been considered as features in the website fingerprinting literature.

We extend the n-gram representation to traffic bursts. Bursts are sequences of consecutive packets in the same direction (either incoming or outgoing). Bursts correlate with the number and order of resources embedded in the page. Additionally, they are more robust to small changes in order than individual sizes because they aggregate several records in the same direction. We represent n-grams of bursts by adding lengths of packets in a direction inside the tuple. In the previous example, the burst-length sequence of the trace above is $(-64,121,-33)$ and the burst bi-grams are $(-64,121),(121,-33)$.

We experimented with uni-, bi- and tri-grams for both features types. We observed a marginal improvement in the classifier on using tri-grams at a substantial cost on the memory requirements of the classifier. We also experimented with the timing of packets. As in website fingerprinting [30], we found that it does not provide reliable prediction power. This is because timing greatly varies depending on the state of the network and thus is not a stable feature to fingerprint web pages. In our experiments, we use the concatenation of uni-grams and bi-grams of both TLS record sizes and bursts as feature set.

Algorithm selection. After experimenting with different supervised classification algorithms, we selected Random Forests (RF) which are known to be very effective for traffic analysis tasks $[18,65]$.

Random forests (RF) are ensembles of simpler classifiers called decision trees. Decision trees use a tree data structure to represent splits of the data: nodes represent a condition on one of the data features and branches represent decisions based on the evaluation of that condition. In decision trees, feature importance in classification is measured with respect to how well they split samples with respect to the target classes. The more skewed the distribution of samples into classes is, the better the feature discriminates. Thus, a common metric for importance is the Shannon's entropy of this distribution. Decision trees, however, do not generalize well and tend to overfit the training data [66]. RFs mitigate this issue by randomizing the data and features over a large amount of trees, using different subsets of features and data in each tree. The final decision of the RF is an aggregate function on the individual decisions of its trees. In our experiments, we use 100 trees and a majority vote to aggregate them.

\footnotetext{
${ }^{4}$ https://github.com/facebookexperimental/doh-proxy

${ }^{5}$ https://git.lekensteyn.nl/peter/wireshark-notes
} 
Validation. We evaluate the effectiveness of DNS fingerprinting in two scenarios typically used in the website fingerprinting literature. A closed world, in which the adversary knows the set of all possible pages users may visit; and an open-world, in which the adversary only has access to a set of monitored pages, and the user may visit pages outside of this set.

In the closed world, we evaluate the effectiveness of our classifier measuring the per-webpage Precision, Recall and F1-Score. We consider positives as DoH traces generated by that webpage and negatives as traces generated by any other webpage. For each webpage, true positives are $\mathrm{DoH}$ traces generated by visits to the webpage that the classifier correctly assigns to that webpage; false positives are traces generated by vists to other pages that are incorrectly classified as the webpage; false negatives are traces of the webpage that are classified as other pages; and true negatives are traces of other pages that are not classified as the webpage. Then, Precision is the ratio of true positives to the total number of traces that were classified as positive (true positives and false positives); Recall is the ratio of true positives to the total number of positives (true positives and false negatives); and the F1-score is the harmonic mean of Precision and Recall. We aggregate these metrics over all the webpages, providing their average and standard deviation.

In the open world there are only two classes: monitored (positive) and unmonitored (negative). Thus, a true positive in the open world is a trace of a monitored webpage that is classified as monitored, and a false positive is a trace of an unmonitored page that is classified as monitored. Likewise, a true negative is a trace of an unmonitored page classified as unmonitored and a false negative a trace of a monitored page classified as unmonitored.

We use 10-fold cross-validation, a standard methodology in machine learning, to measure the generalization error of the classifier, also known as overfitting. In cross-validation, the samples of each class are divided in ten disjoint partitions. The classifier is then trained on each set of nine partitions and tested in the remaining one. Since there are $\left(\begin{array}{c}10 \\ 9\end{array}\right)=10$ possible sets of nine partitions, this provides us ten samples of the classifier performance on a set of samples on which it has not been trained on. Taking the average and standard deviation of these samples gives us an estimate of the performance of the classifier on unseen examples.

\section{B. Evaluating n-grams features}

We now evaluate the effectiveness of n-grams features to launch DNS fingerprinting attacks. We also compare these features with traditional website fingerprinting features in both DoH traffic and web traffic over HTTPS.

Evaluation in closed and open worlds. We first evaluate the attack in a closed world using the LOC1 dataset. We try three settings: i. an adversary that attacks the full dataset of 1,500 websites, ii. an adversary that attacks the curated dataset of 1,414 websites after we eliminate spurious errors, and iii. an adversary that attacks the full dataset but considers regional versions of given pages to be equivalent. For example, classifying google.es as google.co.uk, a common error in our classifier, is considered a true positive. We see in Table II that testing on the clean dataset offers just a $1 \%$ performance increase, and that considering regional versions
Table II: Classifier performance for LOC1 dataset (mean and standard deviation for 10 -fold cross validation).

\begin{tabular}{rccc}
\hline Scenario & Precision & Recall & F1-score \\
\hline Curated traces & $0.914 \pm 0.002$ & $0.909 \pm 0.002$ & $0.908 \pm 0.002$ \\
Full dataset & $0.904 \pm 0.003$ & $0.899 \pm 0.003$ & $0.898 \pm 0.003$ \\
Combined labels & $0.940 \pm 0.003$ & $0.935 \pm 0.003$ & $0.934 \pm 0.003$ \\
\hline
\end{tabular}

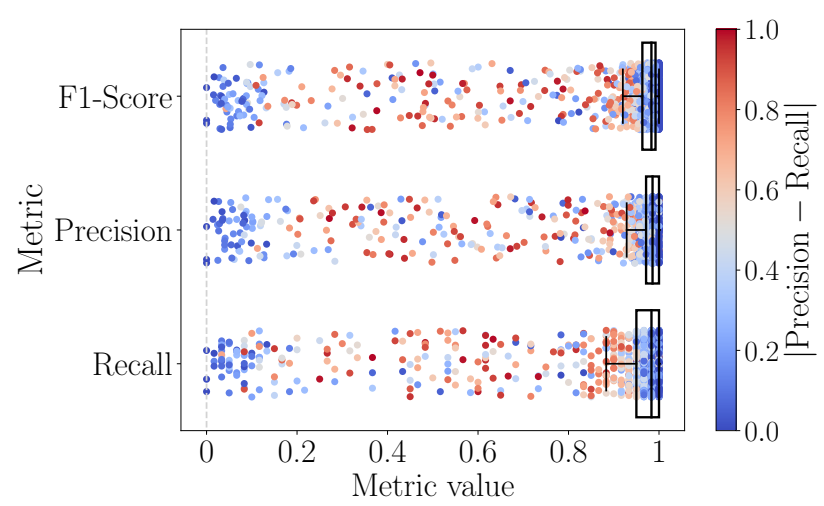

Figure 2: Performance per class in LOC1. Each dot represents a class and its color the absolute difference between Precision and Recall (blue low, red high).

as equivalent results provides an additional 3\% increase. Given the minimal differences, in the remainder of the experiments we use the full dataset.

In the context of website fingerprinting, Overdorf et al. [67] showed that it is likely that the classifier's performance varies significantly between different individual classes. Thus, looking only at average metrics, as in Table II, may give an incomplete and biased view of the classification results. To check if this variance holds on DNS traces we study the performance of the classifier for individual websites. The result is shown in Figure 2. In this scatterplot each dot is a website and its color represents the absolute difference between Precision and Recall: blue indicates 0 difference and red indicates maximum difference (i.e., $\mid$ Precision - Recall $\mid=1$ ). We see that some websites (red dots on the right of the Precision scatterplot) have high Recall - they are often identified by the classifier, but low Precision - other websites are also identified as the website. Thus, these websites have good privacy since the false positives provide the users with plausible deniability. For other pages (red dots on the right of the Recall scatterplot), the classifier obtains low Recall - it almost never identifies them, but high Precision - if they are identified, the adversary is absolutely sure her guess is correct. The latter case is very relevant for privacy, and in particular, censorship, as it enables the censor to block without fear of collateral damage.

Open world. In the previous experiments, the adversary knew that the webpage visited by the victim was within the training dataset. We now evaluate the adversary's capability to distinguish those webpages from other unseen traffic. Following prior work $[65,68]$ we consider two sets of webpages, one monitored and one unmonitored. The adversary's goal is to determine whether a test trace belongs to a page within the monitored set. 


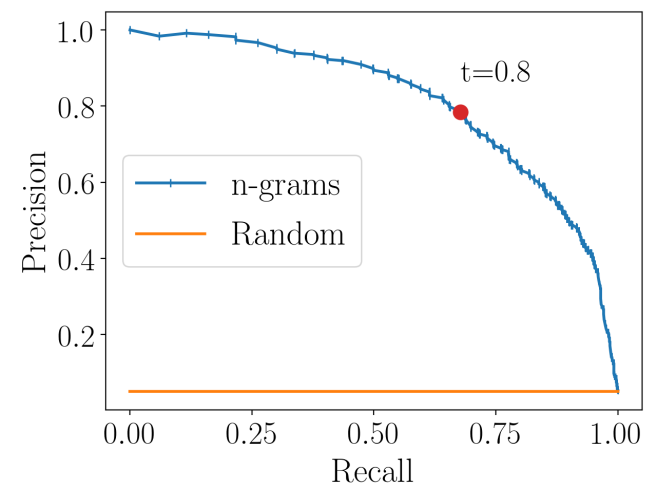

Figure 3: Precision-Recall ROC curve for open world classification, for the monitored class. The notches indicate the variation in threshold, $t$.

We train a classifier with both monitored and unmonitored samples. Since it is not realistic to assume that an adversary can have access to all unmonitored classes, we create unmonitored samples using 5,000 webpages traces formed by a mix of the OW and LOC1 datasets. We divide the classes such that $1 \%$ of all classes are in the monitored set and $10 \%$ of all classes are used for training. We select monitored pages at random. This assumes that different adversaries may be interested in blocking different pages, and enables us to evaluate the average case. We ensure that the training dataset is balanced, i.e., it contains equal number of monitored and unmonitored samples; and the test set contains an equal number of samples from classes used in training and classes unseen by the classifier. When performing cross validation, in every fold, we consider a different combination of the monitored and unmonitored classes for training and testing so that we do not overfit to a particular case.

To decide whether a target trace is monitored or unmonitored, we use a method proposed by Stolerman et al. [69]. We assign the target trace to the monitored class if and only if the classifier predicts this class with probability larger than a threshold $t$, and to unmonitored otherwise. We calculate the Precision-Recall ROC curve for the monitored class using scikit-learn's precision-recall curve plotting function, which varies the discrimination threshold, $t$. The curve is shown in Figure 3, where the notches indicate the varying $t$.

We also plot in Figure 3 the curve corresponding to a random classifier, a naive classifier that outputs positive with probability the base rate occurrence of the positive class. This classifier serves as baseline to assess the effectiveness of the $n$-grams. When we vary the discrimination threshold of the random classifier, Precision remains constant, i.e., the threshold affects TPs and FPs in the same proportion. The effect of the threshold in FNs, however, is inversely proportional to TNs. Thus, Recall changes depending on the classifier's threshold. The AUC (Area Under Curve) for random calssifier is 0.05 , while for the $\mathrm{n}$-grams classifier is 0.81 . When $t=0.8$, the $n$-grams classifier has an F1-score of $\approx 0.7$, indicating that traffic analysis is a true threat to DNS privacy even in the open world scenario.

Comparison to web traffic fingerprinting. To understand the gain DNS fingerprinting provides to an adversary, we compare its effectiveness to that of web traffic fingerprinting. We also evaluate the suitability of $n$-grams and traditional
Table III: F1-Score of the n-grams, k-Fingerprinting, CUMUL and DF features for different subsets of traffic: only DoH traffic (DoH-only), only HTTPS traffic corresponding to web traffic (Web-only) and mixed (DoH+Web).

\begin{tabular}{cccc}
\hline & DoH-only & Web-only & DoH + Web \\
\hline n-grams & 0.87 & 0.99 & 0.88 \\
k-Fingerprinting [18] & 0.74 & 0.95 & 0.79 \\
CUMUL [16] & 0.75 & 0.92 & 0.77 \\
DF [19] $^{6}$ & 0.51 & 0.94 & 0.75 \\
\hline
\end{tabular}

website fingerprinting features to both fingerprinting problems. We compare it to state-of-the-art attacks that use different features: the k-Fingerprinting attack, by Hayes and Danezis [18], that considers a comprehensive set of features used in the website fingerprinting literature; CUMUL, by Panchenko et al. [16] which focuses on packets' lengths and order through cumulative features; and Deep Fingerprinting (DF), an attack based on deep convolutional neural networks [19]. In this comparison, we consider a closed-world of 700 websites (WEB dataset) and use a random forest with the same parameters as classification algorithm. We evaluate the performance of the classifiers on only DoH traffic (DoH-only), only HTTPS traffic corresponding to web content traffic (Web-only), and a mix of the two in order to verify the claim in the RFC that DoH [9] holds great potential to thwart traffic analysis. Table III shows the results.

First, we find that for DNS traffic, due to its chatty characteristics, n-grams provide more than $10 \%$ performance improvement with respect to traditional features. DF would probably achieve higher accuracy if it was trained with more data. To obtain a significant improvement, however, DF requires orders of magnitude more data. Thus, it scales worse than our attack. We also see that the most effective attacks are those made on web traffic. This is not surprising, as the variability of resources' sizes in web traffic contains more information than the small DNS packets. What is surprising is that the n-grams features outperform the traditional features also for website traffic. Finally, as predicted by the standard, if DoH and HTTPS are sent on the same TLS tunnel and cannot be separated, both set of features see a decrease in performance. Still, n-grams outperforms traditional features, with a $10 \%$ improvement.

In summary, the best choice for an adversary with access to the isolated HTTPS flow is to analyse that trace with our novel ngrams features. However, if the adversary is in 'a different path than the communication between the initiator and the recipient' [6] where she has access to DNS, or is limited in resources (see below), the DNS encrypted flow provides comparable results.

Adversary's effort. An important aspect to judge the severity of traffic analysis attacks is the effort needed regarding data collection to train the classifier [28]. We study this effort from two perspectives: amount of samples required - which relates to the time needed to prepare the attack, and volume of data - which relates to the storage and processing requirements for the adversary.

\footnotetext{
${ }^{6}$ We evaluate DF's accuracy following the original paper, i.e., using validation and test sets, instead of 10 -fold cross-validation.
} 
Table IV: Classifier performance for different number of samples in the LOC1 dataset averaged over 10-fold cross validation (standard deviations less than $1 \%$ ).

\begin{tabular}{cccc}
\hline Number of samples & Precision & Recall & F1-score \\
\hline 10 & 0.873 & 0.866 & 0.887 \\
20 & 0.897 & 0.904 & 0.901 \\
40 & 0.908 & 0.914 & 0.909 \\
100 & 0.912 & 0.916 & 0.913 \\
\hline
\end{tabular}

We first look at how many samples are required to train a well-performing classifier. We see in Table IV that there is a small increase between 10 and 20 samples, and that after 20 samples, there are diminishing returns in increasing the number of samples per domain. This indicates that, in terms of number of samples, the collection effort to perform website identification on DNS traces is much smaller than that of previous work on web traffic analysis: Website fingerprinting studies in Tor report more than $10 \%$ increase between 10 and 20 samples [30] and between $2 \%$ and $10 \%$ between 100 and 200 samples [53, 19].

We believe the reason why fingerprinting DoH requires fewer samples per domain is DoH's lower intra-class variance with respect to encrypted web traffic. This is because sources of large variance in web traffic, such as the presence of advertisements which change accross visits thereby varying the sizes of the resources, does not show in DNS traffic for which the same ad-network domains are resolved [70].

In terms of volume of data required to launch the attacks, targeting DoH flows also brings great advantage. In the WEB dataset, we observe that web traces have a length of 1.842 $\mathrm{MB} \pm 2.620 \mathrm{MB}$, while their corresponding DoH counterpart only require $0.012 \mathrm{MB} \pm 0.012 \mathrm{MB}$. While this may not seem a significant difference, when we look at the whole dataset instead of individual traces, the HTTPS traces require $73 \mathrm{~GB}$ while the DoH-only dataset fits in less than $1 \mathrm{~GB}(0.6 \mathrm{~GB})$. This is because DNS responses are mostly small, while web traffic request and responses might be very large and diverse (e.g., different type of resources, or different encodings).

In our experiments, to balance data collection effort and performance, we collected 60 samples per domain for all our datasets. For the unmonitored websites in the open world we collected just three samples per domain (recall that we do not identify unmonitored websites).

\section{DNS Fingerprinting Robustness}

In practice, the capability of the adversary to distinguish websites is very dependent on differences between the setup for training data collection and the environmental conditions at attack time [71]. We present experiments exploring three environmental dimensions: time, space, and infrastructure.

1) Robustness over time: DNS traces vary due to the dynamism of webpage content and variations in DNS responses (e.g., service IP changes because of load-balancing). To understand the impact of this variation on the classifier, we collect data LOC1 for 10 weeks from the end of September to the beginning of November 2018. We divide this period into five intervals, each containing two consecutive weeks, and report in Table $\mathrm{V}$ the F1-score of the classifier when we train the
Table V: F1-score when training on the interval indicated by the row and testing on the interval in the column (standard deviations less than $1 \%$ ). We use 20 samples per webpage (the maximum number of samples collected in all intervals).

\begin{tabular}{cccccc}
\hline F1-score & 0 weeks old & 2 weeks old & 4 weeks old & 6 weeks old & 8 weeks old \\
\hline 0 weeks old & 0.880 & 0.827 & 0.816 & 0.795 & 0.745 \\
2 weeks old & 0.886 & 0.921 & 0.903 & 0.869 & 0.805 \\
4 weeks old & 0.868 & 0.898 & 0.910 & 0.882 & 0.817 \\
6 weeks old & 0.775 & 0.796 & 0.815 & 0.876 & 0.844 \\
8 weeks old & 0.770 & 0.784 & 0.801 & 0.893 & 0.906 \\
\hline
\end{tabular}

classifier on data from a single interval and use the other intervals as test data ( 0 weeks old denotes data collected in November). In most cases, the F1-score does not significantly decrease within a period of 4 weeks. Longer periods result in significant drops - more than $10 \%$ drop in F1-score when the training and testing are separated by 8 weeks.

This indicates that, to obtain best performance, an adversary with the resources of a university research group would need to collect data at least once a month. However, it is unlikely that DNS traces change drastically. To account for gradual changes, the adversary can perform continuous collection and mix data across weeks. In our dataset, if we combine twoand three-week-old samples for training; we observe a very small decrease in performance. Thus, a continuous collection strategy can suffice to maintain the adversary's performance without requiring periodic heavy collection efforts.

2) Robustness across locations: DNS traces may vary across locations due to several reasons. First, DNS lookups vary when websites adapt their content to specific geographic regions. Second, popular resources cached by resolvers vary across regions. Finally, resolvers and $\mathrm{CDNs}$ use geo-location methods to load-balance requests, e.g., using anycast and EDNS [72, 73].

We collect data in three locations, two countries in Europe (LOC1 and LOC2) and a third country in Asia (LOC3). Table VI (leftmost) shows the classifier performance when crossing these datasets for training and testing. When trained and tested on the same location unsurprisingly the classifier yields results similar to the ones obtained in the base experiment. When we train and test on different locations, the F1score decreases between a $16 \%$ and a $27 \%$, the greatest drop happening for the farthest location, LOC3, in Asia.

Interestingly, even though LOC2 yields similar F1-Scores when cross-classified with LOC1 and LOC3, the similarity does not hold when looking at Precision and Recall individually. For example, training on LOC2 and testing on LOC1 results in around $77 \%$ Precision and Recall, but training on LOC1 and testing on LOC2 gives $84 \%$ Precision and $65 \%$ Recall. Aiming at understanding the reasons behind this asymmetry, we build a classifier trained to separate websites that obtain high recall (top 25\% quartile) and low recall (bottom 25\% quartile) when training with LOC1 and LOC3 and testing in LOC2. A feature importance analysis on this classifier showed that LOC2's low-recall top features have a significantly lower importance in LOC1 and LOC2. Furthermore, we observe that the intersection between LOC1 and LOC3's relevant feature sets is slightly larger than their respective intersections with LOC2. While it is clear that the asymmetry is caused by the 

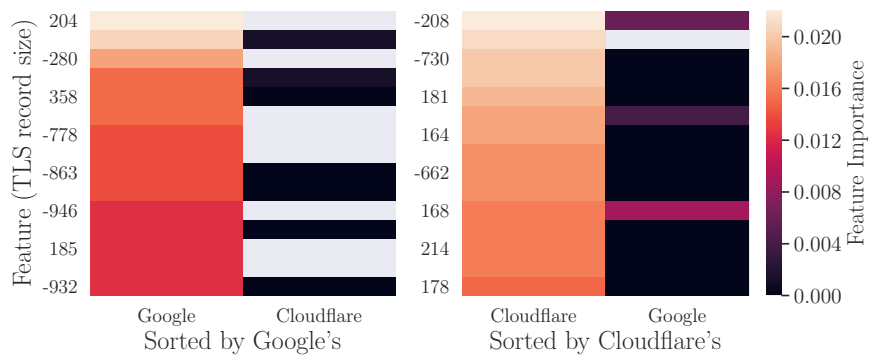

Figure 4: Top 15 most important features in Google's and Cloudflare's datasets. On the left, features are sorted by the results on Google's dataset and, on the right, by Cloudflare's.

configuration of the network in LOC2, its exact cause remains an open question.

3) Robustness across infrastructure: In this section, we study how the DoH resolver and client, and the user platform affect influence the attack's performance.

Influence of DoH Resolver. We study two commercial DoH resolvers, Cloudflare's and Google's. Contrary to Cloudflare, Google does not provide a stand-alone DoH client. To keep the comparison fair, we instrument a new collection setting using Firefox in its trusted recursive resolver configuration with both DoH resolvers.

Table VI (center-left) shows the result of the comparison. As expected, training and testing on the same resolver yields the best results. As in the location setting, we observe an asymmetric decrease in one of the directions: training on GOOGLE dataset and attacking CLOUD results in 13\% F1-score, while attacking GOOGLE with a classifier trained on CLOUD yields similar results as training on GOOGLE itself.

To investigate this asymmetry we rank the features according their importance for the classifiers. For simplicity, we only report the result on length unigrams, but we verified that our conclusions hold when considering all features together. Figure 4 shows the top-15 most important features for a classifier trained on Google's resolver (left) and Cloudflare's (right). The rightmost diagram of each column shows the importance of these features on the other classifier. Red tones indicate high importance, and dark colors represent irrelevant features. Grey indicates that the feature is not present.

We see that the most important features in Google are either not important or missing in Cloudflare (the right column in left-side heatmap is almost gray). As the missing features are very important, they induce erroneous splits early in the trees, and for a larger fraction of the data, causing the performance drop. However, only one top feature in the classifier trained on Cloudflare is missing in Google, and the others are also important (right column in right-side heatmap). Google does miss important features in Cloudflare, but they are of little importance and their effect on performance is negligible.

Influence of end user's platform. We collect traces for the 700 top Alexa webpages on a Raspberry Pi (RPI dataset) and an Ubuntu desktop (DESKTOP dataset), both from LOC1. We see in Table VI (center-right) that, as expected, the classifier has good performance when the training and testing data come from the same platform. However, it drops to almost zero when crossing the datasets.
When taking a closer look at the TLS record sizes from both platforms we found that TLS records in the DESKTOP dataset are on average 7.8 bytes longer than those in RPI (see Figure 12 in Appendix D). We repeated the cross classification after adding 8 bytes to all RPI TLS record sizes. Even though the classifiers do not reach the base experiment's performance, we see a significant improvement in cross-classification F1-score to 0.614 when training on DESKTOP and testing on RPI, and 0.535 when training on RPI and testing on DESKTOP.

Influence of DNS client. Finally, we consider different client setups: Firefox's trusted recursive resolver or TRR (CLOUD), Cloudlflare's DoH client with Firefox (CL-FF) and Cloudflare's DoH client with Chrome (LOC2). We collected these datasets in location LOC2 using Cloudflare's resolver.

Table VI (rightmost) shows that the classifier performs as expected when trained and tested on the same client setup. When the setup changes, the performance of the classifier drops dramatically, reaching zero when we use different browsers. We hypothesize that the decrease between CL-FF and LOC2 is due to differences in the implementation of the Firefox's built-in and Cloudflare's standalone DoH clients.

Regarding the difference when changing browser, we found that Firefox' traces are on average 4 times longer than Chrome's. We looked into the unencrypted traffic to understand this difference. We used the man-in-the-middle proxy and the Lekensteyn's scripts to decrypt DoH captures for Firefox configured to use Cloudflare's resolver. For Google's resolver, we man-in-the-middle a curl-doh client ${ }^{7}$, which also has traces substantially shorter than Firefox. We find that Firefox, besides resolving domains related to the URL we visit, also issues resolutions related to OSCP servers, captive portal detection, user's profile/account, web extensions, and other Mozilla servers. As a consequence, traces in CL-FF and CLOUD datasets are substantially larger and contain different TLS record sizes than any of our other datasets. We conjecture that Chrome performs similar requests, but since traces are shorter we believe the amount of checks seems to be smaller than Firefox's.

4) Robustness Analysis Takeaways: Our robustness study shows that to obtain best results across different configurations the adversary needs i) to train a classifier for each targeted setting, and ii) to be able to identify her victim's configuration. Even if the adversary needs to train a classifier for each setting, this is less costly in the case of DoH fingerprinting as compared to website fingerprinting due to the training requiring significantly less data. Kotzias et al. demonstrated that identifying client or resolver is possible, for instance examining the IP (if the IP is dedicated to the resolver), or fields in the ClientHello of the TLS connection (such as the Server Name Indication (SNI), cipher suites ordering, etc.) [74]. Even if TLS 1.3 encrypts some of these headers and are thus not directly available to the adversary, we found that characteristics of traffic itself are enough to identify a resolver. We built classifiers to distinguish resolver and client based on the TLS record length. We can identify resolvers with $95 \%$ accuracy, and we get no errors (100\% accuracy) when identifying the client.

When analyzing traces, we observed customization by the DNS providers that are not part of the standard. For example,

\footnotetext{
${ }^{7}$ https://github.com/curl/doh
} 
Table VI: Performance variation changes in location and infrastructure (F1-score, standard deviations less than 2\%).

\begin{tabular}{cccc}
\hline Location & LOC1 & LOC2 & LOC3 \\
\hline LOC1 & 0.906 & 0.712 & 0.663 \\
LOC2 & 0.748 & 0.908 & 0.646 \\
LOC3 & 0.680 & 0.626 & 0.917 \\
\hline
\end{tabular}

\begin{tabular}{ccc}
\hline Resolver & GOOGLE & CLOUD \\
\hline GOOGLE & 0.880 & 0.129 \\
CLOUD & 0.862 & 0.885 \\
\hline
\end{tabular}

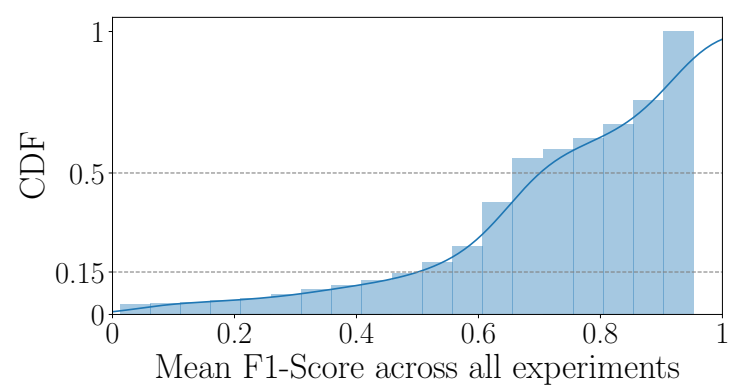

Figure 5: Cumulative Distribution Function (CDF) of the perclass mean F1-Score.

Cloudflare includes HTTP headers such as CF-RAY to trace a request through its network. Such customizations can lead to differences in the traffic among different providers and have an impact on the classification.

Regarding users' platform, we see little difference between desktops, laptops, and servers in Amazon Web Services. However, we observe a difference between these and constrained devices, such a Raspberry Pi. This different results on a drop in accuracy when training a classifier on one type and deploying it on the other.

Finally, our longitudinal analysis reveals that, keeping up with the changes in DNS traces can be done at low cost by continuously collecting samples and incorporating them to the training set.

Survivors and Easy Preys. We study whether there are websites that are particularly good or bad at evading fingerprinting under any circumstance. We compute the mean F1Score across all configurations as an aggregate measure of the attack's overall performance. We plot the CDF of the distribution of mean F1-scores over the websites in Figure 5. This distribution is heavily skewed: there are up to $15 \%$ of websites that had an F1-Score equal or lower than 0.5 and more than $50 \%$ of the websites have a mean F1-Score equal or lower than 0.7 .

We rank sites by lowest mean F1-Score and lowest standard deviation. At the top of this ranking, there are sites that survived the attack in all configurations. Among these survivors, we found Google, as well as sites giving errors, that get consistently misclassified as each other. We could not find any pattern in the website structure or the resource loads that explains why other sites with low F1 survive. At the bottom of the ranking, we find sites with long domain names, with few resources and that upon visual inspection present low variability.

Data Pollution Our experiments evaluate the scenario where a user visits a single webpage at a time. However, in many cases the user might visit more than one page at a time, might have multiple tabs open, or might have applications running in the background. These scenarios could pollute the traces

\begin{tabular}{ccc}
\hline Platform & DESKTOP & RPI \\
\hline DESKTOP & 0.8802 & 0.0003 \\
RPI & 0.0002 & 0.8940 \\
\hline
\end{tabular}

\begin{tabular}{cccc}
\hline Client & CLOUD & CL-FF & LOC2 \\
\hline CLOUD & 0.885 & 0.349 & 0.000 \\
CL-FF & 0.109 & 0.892 & 0.069 \\
LOC2 & 0.001 & 0.062 & 0.908 \\
\hline
\end{tabular}

by mixing the DoH traffic from multiple webpages. Since it is non-trivial for an adversary to split traffic belonging to different webpages, such pollution can have an impact on the attack effectiveness. However, at the time of writing there is no standard way in the literature to study the impact of polluted DNS traffic. Designing a method to evaluate this effect is beyond the scope of this work.

Pollution could also be triggered on the resolver side, if the resolver serves both $\mathrm{DoH}$ and non-DoH traffic on the same connection. The resolvers studied in our experiments, Cloudflare and Google, only host one webpage: one.one. one. one and dns.google.com, respectively. By analyzing the traffic of a user visiting these webpages, we observe that the TLS flows carrying the web content and the DoH flows corresponding to the domain resolution can be trivially distinguished using traffic features such as the number of packets (HTTPS traces are longer than DoH traces) and the packet sizes (DNS responses are much smaller than the resources sent over HTTPS). We do not expect this kind of pollution to be an obstacle for the adversary.

\section{DNS DEFENSES AGAINST FINGERPRINTING}

In this section, we evaluate existing techniques to protect encrypted DNS against traffic analysis. Table VII summarizes the results. We consider the following defenses:

EDNS(0) Padding. EDNS (Extension mechanisms for DNS) is a specification to increase the functionality of the DNS protocol [75]. It specifies how to add padding [10], both on DNS clients and resolvers, to prevent size-correlation attacks on encrypted DNS. The recommended padding policy is for clients to pad DNS requests to the nearest multiple of 128 bytes, and for resolvers to pad DNS responses to the nearest multiple of 468 bytes [76].

Cloudflare's DoH client provides functionality to set $\operatorname{EDNS}(0)$ padding to DNS queries, leaving the specifics of the padding policy to the user. We modify the client source code to follow the padding strategy above. Google's specification also mentions EDNS padding. However, we could not find any option to activate this feature, thus we cannot analyze it.

In addition to the EDNS(0) padding, we wanted to see whether simple user-side measures that alter the pattern of requests, such as the use of an ad-blocker, could be effective countermeasures. We conduct an experiment where we use Selenium with a Chrome instance with the AdBlock Plus extension installed. We do this with DoH requests and responses padded to multiples of 128 bytes.

Upon responsible disclosure of this work, Cloudflare's added padding to the responses of their DoH resolver. However, when analyzing the collected data we discover that they do not follow the recommended policy. Instead of padding to multiples of 468 bytes, Cloudflare's resolver pads responses to multiples of 128 bytes, as recommended for DoH clients. 
In order to also evaluate the recommended policy, we set up an HTTPS proxy (mitmproxy) between the DoH client and the Cloudflare resolver. The proxy intercepts responses from Cloudflare's DoH resolver, strips the existing padding, and pads responses to the nearest multiple of 468 bytes.

As we show below, none of these padding strategies completely stops traffic analysis. To understand the limits of protection of padding, we simulate a setting in which padding is perfect, i.e., all records have the same length and the classifier cannot exploit the TLS record size information. To simulate this setting, we artificially set the length of all packets in the dataset to 825 , the maximum size observed in the dataset.

DNS over Tor. Tor is an anonymous communication network. To protect the privacy of its users, Cloudflare set up a DNS resolver that can be accessed using Tor. This enables users to not reveal their IP to the resolver when doing lookups. To protect users' privacy, Tor re-routes packets through socalled onion routers to avoid communication tracing based on IP addresses; and it packages content into constant-size cells to prevent size-based analysis. These countermeasures have so far not been effective to protect web traffic $[16,17,18,19]$. We study whether they can protect DNS traffic.

Results. Our first observation is that EDNS0 padding is not as effective as expected. Adding more padding, as recommended in the specification, does provide better protection, but still yields an F1-score of 0.45 , six orders of magnitude greater than random guessing. Interestingly, usage of an ad-blocker helps as much as increasing the padding, as shown by the EDNS0-128-adblock experiment. As shown below, Perfect Padding would actually deter the attack, but at a high communication cost.

As opposed to web traffic, where website fingerprinting obtains remarkable performance [61, 18, 19], Tor is very effective in hiding the websites originating a DNS trace. The reason is that DNS lookups and responses are fairly small. They fit in one, at most two, Tor cells which in turn materialize in few observed TLS record sizes. As a result, it is hard to find features unique to a page. Also, DNS traces are shorter than normal web traffic, and present less variance. Thus, length-related features, which have been proven to be very important in website fingerprinting, only provide a weak $1 \%$ performance improvement.

Even though Perfect Padding and DNS over Tor offer similar performance, when we look closely at the misclassified webpages, we see that their behavior is quite different. For Tor, we observe misclassifications cluster around six different groups, and in Perfect Padding they cluster around 12 (different) groups (see the Appendix for an extended version of the paper that includes confusion graphs). For both cases, we tested that it is possible to build a classifier that identifies the cluster a website belongs to with reasonable accuracy. This means that despite the large gain in protection with respect to $\operatorname{EDNS}(0)$, the effective anonymity set for a webpage is much smaller than the total number of webpages in the dataset.

Finally, we evaluate defenses' communication overhead. For each countermeasure, we collect 10 samples of 50 webpages, with and without countermeasures, and measure the difference in total volume of data exchanged between client and resolver.
Table VII: Classification results for countermeasures.

\begin{tabular}{cccc}
\hline Method & Precision & Recall & F1-score \\
\hline EDNS0-128 & $0.710 \pm 0.005$ & $0.700 \pm 0.004$ & $0.691 \pm 0.004$ \\
EDNS0-128-adblock & $0.341 \pm 0.013$ & $0.352 \pm 0.011$ & $0.325 \pm 0.011$ \\
EDNS0-468 & $0.452 \pm 0.007$ & $0.448 \pm 0.006$ & $0.430 \pm 0.007$ \\
Perfect Padding & $0.070 \pm 0.003$ & $0.080 \pm 0.002$ & $0.066 \pm 0.002$ \\
DNS over Tor & $0.035 \pm 0.004$ & $0.037 \pm 0.003$ & $0.033 \pm 0.003$ \\
\hline DNS over TLS & $0.419 \pm 0.008$ & $0.421 \pm 0.007$ & $0.395 \pm 0.007$ \\
\hline
\end{tabular}

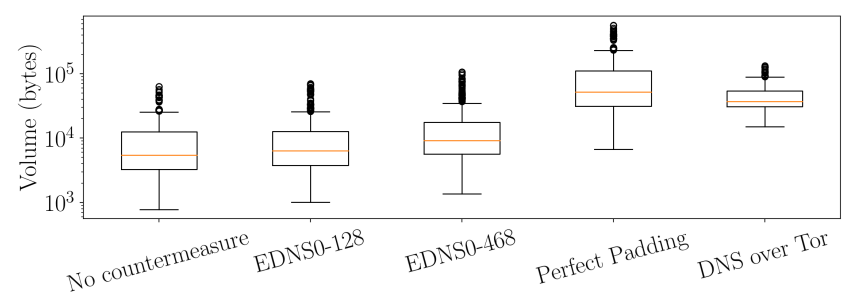

Figure 6: Total traffic volume with and without defenses.

We see in Figure 6 that, as expected, EDNS0 padding (both 128 and 468) incur the least overhead, but they also offer the least protection. DNS over Tor, in turn, offers lower overhead than Perfect Padding.

Comparison with DNS over TLS (DoT). Finally, we compare the protection provided by DNS over HTTPS and over TLS, using the DOT dataset. As in DoH, Cloudflare's DoT resolver implements EDNS0 padding of DNS responses to a multiple of 128 bytes. The cloudflared DoT client, however, does not support DoT traffic. Thus, we use the Stubby client to query Cloudflare's DoT padded to a multiple of 128 bytes.

Our results (shown in the last row of Table VII) indicate that DoT offers much better protection than DoH - about 0.3 reduction in F1-score. We plot a histogram of sizes of the sent and received TLS records for both DoH and DoT for 100 webpages (Figure 7). We observe that DoT traffic presents much less variability in TLS record sizes than DoH.

To gain insights into the origin of this variability, we collected and decrypted DoT and DoH traffic for visits to a handful of websites. We used a modified Stubby client ${ }^{8}$ to decrypt the DoT traffic, and used the tools described in Section V-A to decrypt the DoH traces. For each website, we used Firefox and Stubby (DoT setting) and, immediately after finishing loading the page, we restarted Firefox with a clean profile and visited using Firefox's native DoH client (DoH setting).

Upon inspection of the traces, we observe that DoH traces contain requests for $\mathrm{A}$ and AAAA records while DoT traces only have requests for A records. This shows how different implementations of the client can generate significantly different traffic, potentially affecting the performance of the attack. This also explains why we observe a greater number of TLS record sizes in Figure 7. We also find differences in the domains that are resolved. However, most of the differences are domains that occur independently of the protocol and, thus, cannot explain the difference in attack performance between the two protocols. The most relevant difference is that, excluding AAAA queries and responses, DoT traces have

\footnotetext{
${ }^{8}$ https://github.com/saradickinson/getdns/tree/1.5.2_add_keylogging
} 
fewer average number of DNS messages than DoH traces for the same website. In addition, the TLS records in DoT traces are larger than DoH's 9 . We acknowledge that although we identify possible causes for the variability of DoH's TLS record sizes, our observations are not conclusive on whether this variability accounts for the better performance of the attack on DoT than DoH.
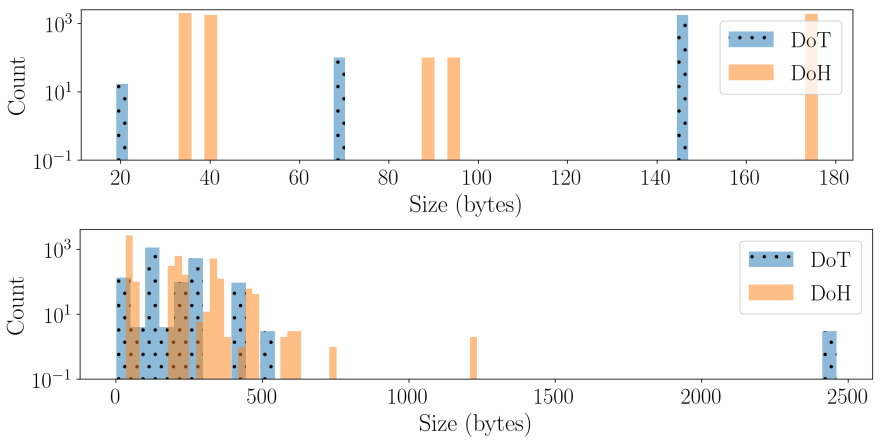

Figure 7: Histogram of sent (top) and received (bottom) TLS record sizes for DoT and DoH.

\section{CENSORSHIP ON ENCRYPTED DNS}

DNS-based blocking is a wide-spread method of censoring access to web content. Censors inspect DNS lookups and, when they detect a blacklisted domain, they either reset the connection or inject their own DNS response [77]. The use of encryption in DoH precludes content-based lookup identification. The only option left to the censor is to block the resolver's IP. While this would be very effective, it is a very aggressive strategy, as it would prevent users from browsing any web. Furthermore, some DoH resolvers, such as Google's, do not necessarily have a dedicated IP. Thus, blocking their IP may also affect other services.

An alternative for the censor is to use traffic analysis to identify the domain being looked up. In this section, we study whether such an approach is feasible. We note that to block access to a site, a censor not only needs to identify the lookup from the encrypted traffic, but also needs to do this as soon as possible to prevent the user from downloading any content.

\section{A. Uniqueness of DoH traces}

In order for the censor to be able to uniquely identify domains given DoH traffic, the DoH traces need to be unique. In particular, to enable early blocking, the first packets of the trace need to be unique.

To study the uniqueness of DoH traffic, let us model the set of webpages in the world as a random variable $W$ with sample space $\Omega_{W}$; and the set of possible network traces generated by those websites as a random variable $S$ with sample space $\Omega_{S}$. A website's trace $w$ is a sequence of non-zero integers: $\left(s_{i}\right)_{i=1}^{n}, s_{i} \in \mathbb{Z} \backslash\{0\}, n \in \mathbb{N}$, where $s_{i}$ represents the size (in bytes) of the $i$-th TLS record in the traffic trace. Recall that its sign represents the direction - negative for incoming (DNS to client) and positive otherwise. We denote partial traces, i.e., only the $l$ first TLS records, as $S_{l}$.

\footnotetext{
${ }^{9}$ This might seem counterintuitive as DoH has HTTP headers that DoT does not have. However, DoH's RFC allows the use of HTTP compression (and we observe its use in our dataset), while DoT's RFC recommends against the use of TLS compression.
}

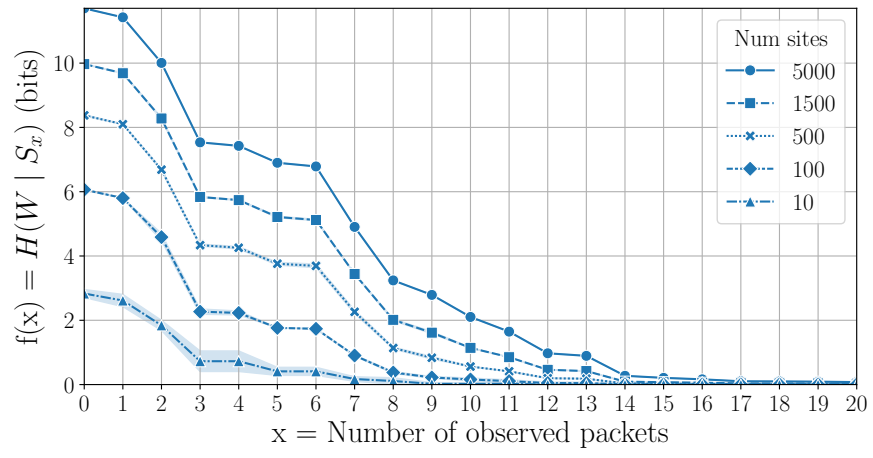

Figure 8: Conditional entropy $H\left(W \mid S_{l}\right)$ given partial observations of DoH traces for different world sizes $\left(\left|\Omega_{W}\right|=\{10,100,500,1500,5000\}\right)$. Each data point is averaged over 3 samples.

We measure uniqueness of partial traces using the conditional entropy $H\left(W \mid S_{l}\right)$, defined as:

$$
H\left(W \mid S_{l}\right)=\sum_{\forall o \in \Omega_{S_{l}}} \operatorname{Pr}\left[S_{l}=o\right] H\left(W \mid S_{l}=o\right) .
$$

Here, $H\left(W \mid S_{l}=o\right)$ is the Shannon entropy of the probability distribution $\operatorname{Pr}\left[W \mid S_{l}=o\right]$. This probability describes the likelihood that the adversary guesses websites in $W$ given the observation $o$. The conditional entropy $H\left(W \mid S_{l}\right)$ measures how distinguishable websites in $\Omega_{W}$ are when the adversary has only observed $l$ TLS records. When this entropy is zero, sites are perfectly distinct. For instance, if the first packet of every DoH trace had a different size, then the entropy $H(W \mid$ $S_{1}$ ) would be 0 .

We compute the conditional entropy for different world sizes $\left|\Omega_{W}\right|$ and partial lengths $l$, using traces from the OW dataset. We show the result in Figure 8. Every point is an average over 3 samples of $\left|\Omega_{W}\right|$ webs. These webs are selected uniformly at random from the dataset. The shades represent the standard deviation across the 3 samples.

Unsurprisingly, as the adversary observes more packets, the traces become more distinguishable and the entropy decreases. For all cases, we observe a drop of up to 4 bits within the first four packets, and a drop below 0.1 bits after 20 packets. As the world size increases, the likelihood of having two or more websites with identical traces increases, and thus we observe a slower decay in entropy. We also observe that, as the world size increases the standard deviation becomes negligible. This is because the OW dataset contains 5,000 websites. Thus, as the size of the world increases, the samples of $\Omega_{W}$ contain more common websites.

Even when considering 5,000 pages, the conditional entropy drops below 1 bit after 15 packets. This means that after 15 packets have been observed, there is one domain whose probability of having generated the trace is larger than 0.5 . In our dataset 15 packets is, on average, just $15 \%$ of the whole trace. Therefore, on average, the adversary only needs to observe the initial $15 \%$ of a DoH connection to determine a domain with more confidence than taking a random guess between two domains. We observe that as the number of pages grows, the curves are closer to each other indicating 

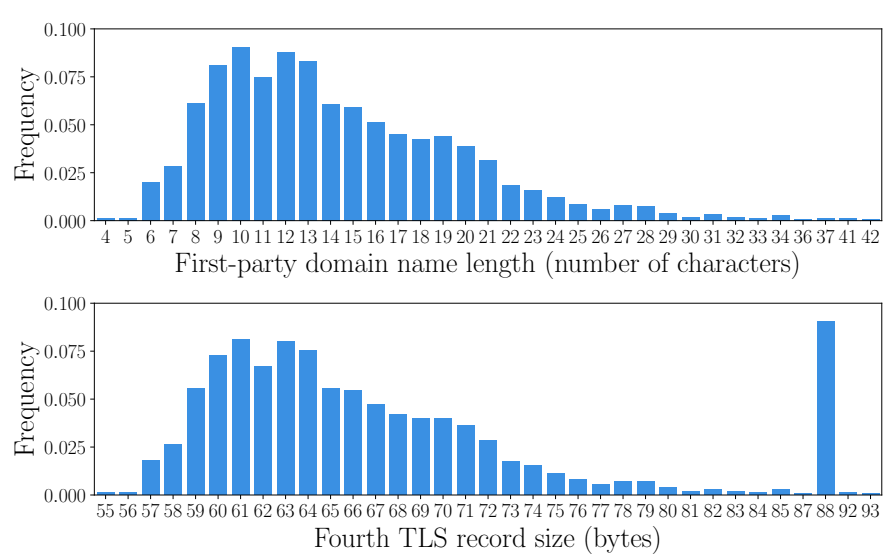

Figure 9: Histograms for domain name length (top) and fourth TLS record length (bottom) in the LOC1 dataset, for 10 samples (normalized over the total sum of counts).

that convergence on uniqueness after the 15-th packet is likely to hold in larger datasets.

The importance of the fourth packet. We hypothesized that the large drop by the fourth packet might be caused by one of these records containing the DNS lookup. As these traces do not contain padding, the domain length would be directly observable in the trace. We verify this hypothesis by comparing the frequency of appearance of the domain's and outgoing fourth record's length (incoming records cannot contain a lookup). We discard TLS record sizes corresponding to HTTP2 control messages, e.g., the size "33" which corresponds to HTTP2 acknowledgements and outliers that occurred $5 \%$ or less times. However, We kept size "88" even though it appears too often, as it could be caused by queries containing 37 characters-long domain names.

We represent the frequency distributions in Figure 9. We see that the histogram of the sizes of the fourth TLS record in our dataset is almost identical to the histogram of domain name lengths, being the constant difference of 51 bytes between the two histograms the size of the HTTPS header. This confirms that the fourth packet often contains the first-party DoH query. In some traces the DoH query is sent earlier, explaining the entropy decrease starting after the second packet.

\section{B. Censor DNS-blocking strategy}

We study the collateral damage, i.e., how many sites are affected when a censor blocks one site after a traffic-analysis based inference. We assume that upon decision, the censor uses standard techniques to block the connection [78].

High-confidence blocking. To minimize the likelihood of collateral damage, the adversary could wait to see enough packets for the conditional entropy to be lower than one bit. This requires waiting for, on average, $15 \%$ of the TLS records in the DoH connection before blocking. As those packets include the resolution to the first-party domain, the client can download the content served from this domain. Yet, the censor can still disrupt access to subsequent queried domains (subdomains and third-parties). This is a strategy already used in the wild as a stealthy form of censorship [78].

To avoid this strategy, the clients could create one connection per domain lookup, thereby mixing connections belonging to

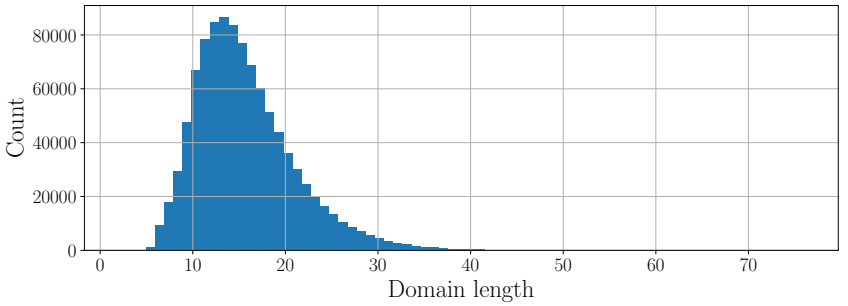

Figure 10: Distribution of domain length in the Alexa top 1M.

Table VIII: Number of domains in each censorship test list and their presence in the Alexa top 1M.

\begin{tabular}{lccccc}
\hline & Turkmenistan & Iran & S. Arabia & Vietnam & China \\
\hline Censored domains & 344 & 877 & 284 & 274 & 191 \\
Not in Alexa-1M & 246 & 600 & 219 & 218 & 94 \\
\hline
\end{tabular}

the censored page and others. At the cost of generating more traffic for users and resolvers, this would force the censor to drop all DoH connections originating from a user's IP or throttle their DoH traffic, causing more collateral damage.

Block on first DoH query. A more aggressive strategy is to drop the DoH connection before the first $\mathrm{DoH}$ response arrives. While this guarantees that the client cannot access any content, not even index. html, it also results in all domains with same name length being censored.

In order to understand the collateral damage incurred by domain length based blocking relying on the fourth packet, we compare the distribution of domain name lengths in the Alexa top $1 \mathrm{M}$ ranking (see Fig. 10) with the distribution of domain names likely to be censored in different countries. For the former we take the global ranking as per-country rankings only provide 500 top websites which are not enough for the purpose of our experiments and, for some countries, the lists are not even available. We take the test lists provided by Citizen Labs [79]. These lists contain domains that regional experts identify as likely to be censored. While appearance in these lists does not guarantee that the domains are actually censored, we believe that they capture potential censor behavior.

We take five censorship-prone countries as examples: Turkmenistan, Iran, Saudi Arabia, Vietnam, and China. Our analysis of collateral damage is relative among the countries considered in our study. Since the Alexa list contains only domains, we extract the domains from the URLs appearing in Citizen Labs' test lists. Table VIII shows the total number of domains in each list and the number of those domains that are not present in the Alexa-1M list. We observe that at most 51\% (China) of the domains appear in the ranking. For the other countries the ratio is between $21 \%$ and $32 \%$. This indicates that the potentially censored domains themselves are mostly not popular.

Even if the censor blocks unpopular domains, there are two side effects of blocking based on domain lengths. First, there will be some collateral damage: allowed websites with the same domain length will also be blocked. Second, there will be a gain for the censor: other censored websites with the same domain length are blocked at the same time.

We study the trade-off between collateral damage and censor gain. The minimum collateral damage is attained when the 


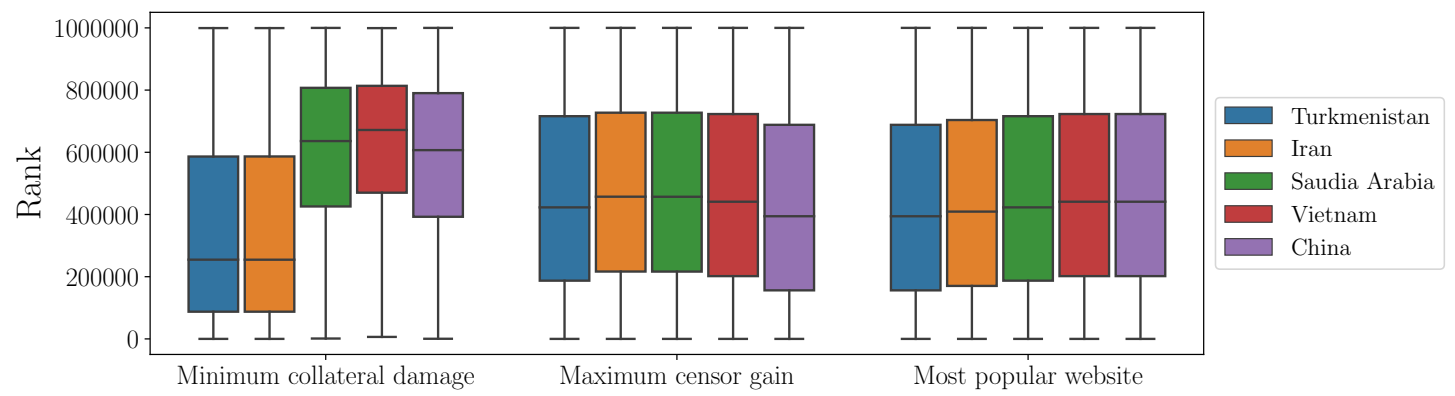

Figure 11: Collateral damage in terms of ranking for three blocking strategies: minimum collateral damage set size, maximum censor gain, most popular website.

censor blocks domains of length 6 (such as $\mathrm{ft}$. com), resulting on 1,318 affected websites. The maximum damage happens when censoring domains of size 10 (such as google.com) which affects other 66,923 websites, domains of size 11 (such as youtube.com) which affects other 78,603 websites, domains of size 12 (such as facebook.com) which affects other 84,471 websites, domains of size 13 (such as wikipedia.org) which affects other 86,597 websites, and domains of size 14 (such as torproject.org, which affects other 83,493 websites. This means that, in the worst case, collateral damage is at most $8.6 \%$ of the top $1 \mathrm{M}$ list.

To understand the censor gain, we consider Iran as an example. The maximum gain is 97 , for domain length of 13 , i.e., when the censor blocks domains of length 13, it blocks 97 domains in the list. At the same time, it results in large collateral damage (86,557 websites). The minimum gain is obtained for domain length 5 , which only blocks one website, but causes small collateral damage (1,317 domains). If Iran blocks the most popular domain in the list (google.com), this results in a collateral damage of 66,887 websites.

The volume of affected websites is of course representative of damage, but it is not the only factor to take into account. Blocking many non-popular domains that are in the bottom of the Alexa list is not the same as blocking a few in the top-100. We show in Figure 11 boxplots representing the distribution of Alexa ranks for three blocking strategies: minimum collateral damage, maximum censor gain, and blocking the most popular web. For each country, these strategies require blocking different domain lengths. Thus, in terms of volume, the damage is different but in the order of the volumes reported above. We observe that the minimum collateral damage strategy results in different impact depending on the country. Although for all of them the volume is much lower than for the other strategies, in Turkmenistan and Iran the median rank of the blocked websites is much lower than those in the other strategies, indicating that this strategy may have potentially higher impact than blocking more popular or high-gain domains. On the positive side, minimum collateral damage in Saudi Arabia, Vietnam, and China, mostly affects high-ranking websites. Thus, this strategy may be quite affordable in this country. The other two strategies, maximum gain and blocking the most popular website, result on a larger number of websites blocked, but their median ranking is high (above 500,000) and thus can also be considered affordable.

In conclusion, our study shows that while block on first DoH query is an aggressive strategy, it can be affordable in terms of collateral damage. More research is needed to fine-tune our analysis, e.g., with access to large per-country rankings, or exact lists of blacklisted domains.

\section{LOOKING AHEAD}

We have shown that, although it is a great step for privacy, encrypting DNS does not completely prevent monitoring or censorship. Current padding strategies have great potential to prevent censorship, but our analysis shows that they fall short when it comes to stopping resourceful adversaries from monitoring users' activity on the web.

Our countermeasures analysis hints that the path towards full protection is to eliminate size information. In fact, the repacketization in constant-size cells offered by Tor provides the best practical protection. Tor, however, induces a large overhead both in the bandwidth required to support onion encryption, as well as in download time due to the rerouting of packets through the Tor network.

We believe that the most promising approach to protect $\mathrm{DoH}$ is to have clients mimicking the repacketization strategies of Tor, without replicating the encryption scheme or re-routing. This has the potential to improve the trade-off between overhead and traffic analysis resistance. A complementary strategy to ease the achievement of constant-size flows, is to rethink the format of DNS queries and its headers. Reducing the number of bits required for the headers would make it easier to fit queries and responses in one constant-size packet with small overhead.

Besides protection against third party observers, it is important that the community also considers protection from the resolvers. The current deployment of $\mathrm{DoH}$, both at the resolvers and browsers, concentrates all lookups among a small number of actors that can observe the behavior of users. More research in the direction of Oblivious DNS [80] is needed to ensure that no parties can become main surveillance actors.

\section{ACKNOWLEDGMENT}

We would like to thank the Cloudflare team, Sara Dickinson, and Daniel Kahn Gillmor, for their collaboration and support on this work. We would also like to thank our shepherd, Zhou Li, for his feedback that helped improve the paper. This research is supported by the Spanish grant TIN2017- 88749-R (DiscoEdge) and by the Research Council KU Leuven under the grant C24/18/049. In addition, this work has been partially supported by the United States Air Force and DARPA under Contract No. FA8750-19-C-0502. Any opinions, findings and conclusions or recommendations expressed in this material are 
those of the authors and do not necessarily reflect the views of the United States Air Force and DARPA. Marc Juarez was supported by a PhD fellowship of the Fund for Scientific Research - Flanders (FWO).

\section{REFERENCES}

[1] Stephane Bortzmeyer. DNS privacy considerations. 2015.

[2] Christian Grothoff, Matthias Wachs, Monika Ermert, and Jacob Appelbaum. NSA's MORECOWBELL: Knell for DNS. Unpublished technical report, 2017.

[3] The NSA files decoded. https://www.theguardian.com/ us-news/the-nsa-files. Accessed: 2019-05-13.

[4] Earl Zmijewski. Turkish Internet Censorship Takes a New Turn. https://dyn.com/blog/turkish-internet-censorship, 2014. Accessed: 2018-12-06.

[5] Nicholas Weaver, Christian Kreibich, and Vern Paxson. Redirecting dns for ads and profit. In FOCI, 2011.

[6] S. Bortzmeyer. DNS Privacy Considerations. RFC 7626, 2015.

[7] A.Cooper, H. Tschofenig, B. Aboba, J. Peterson, J. Morris, M. Hansen, and R. Smith. Privacy Considerations for Internet Protocols. RFC 6973, 2015.

[8] Z. Hu, L. Zhu, J. Heidemann, A. Mankin, D. Wessels, and P. Hoffman. Specification for DNS over Transport Layer Security (TLS). RFC 7858, RFC Editor, May 2016.

[9] P. Hoffman and P. McManus. DNS Queries over HTTPS (DoH). RFC 8484, RFC Editor, October 2018.

[10] A. Mayrhofer. The edns(0) padding option. RFC 7830, RFC Editor, May 2016.

[11] Google Public DNS over HTTPS (DoH) supports RFC 8484 standard. https://security.googleblog.com/2019/06/ google-public-dns-over-https-doh.html. Accessed: 201904-03.

[12] Cloudflare DNS over HTTPS. https://developers. cloudflare.com/1.1.1.1/dns-over-https/. Accessed: 201805-07.

[13] Selena Deckelmann. dns over https (doh) - testing on beta. https://blog.mozilla.org/futurereleases/2018/09/ 13/dns-over-https-doh-testing-on-beta, 2018. accessed: 2018-12-30.

[14] Mozilla Support. Firefox DNS-over-HTTPS: About the US rollout of DNS over HTTPS. https: //support.mozilla.org/en-US/kb/firefox-dns-over-https\# w_about-the-us-rollout-of-dns-over-https. Accessed: 2020-01-07.

[15] Geoff Huston. DOH! DNS over HTTPS explained. https://labs.ripe.net/Members/gih/ doh-dns-over-https-explained, 2018. Accessed: 201812-27.

[16] Andriy Panchenko, Fabian Lanze, Andreas Zinnen, Martin Henze, Jan Pennekamp, Klaus Wehrle, and Thomas Engel. Website fingerprinting at internet scale. In Network \& Distributed System Security Symposium (NDSS), pages 1-15. IEEE Computer Society, 2016.

[17] Tao Wang and Ian Goldberg. On realistically attacking tor with website fingerprinting. In Privacy Enhancing Technologies Symposium (PETS), pages 21-36. De Gruyter Open, 2016.

[18] Jamie Hayes and George Danezis. k-fingerprinting: A robust scalable website fingerprinting technique. In USENIX Security Symposium, pages 1-17. USENIX Association, 2016.
[19] Payap Sirinam, Mohsen Imani, Marc Juarez, and Matthew Wright. Deep fingerprinting: Undermining website fingerprinting defenses with deep learning. In $A C M$ Conference on Computer and Communications Security (CCS), pages 1928-1943. ACM, 2018.

[20] Andrew M. White, Austin R. Matthews, Kevin Z. Snow, and Fabian Monrose. Phonotactic reconstruction of encrypted voip conversations: Hookt on fon-iks. In IEEE Symposium on Security and Privacy (S\&P 2011), 2011.

[21] Se Eun Oh, Shuai Li, and Nicholas Hopper. Fingerprinting keywords in search queries over tor. PoPETs, 2017.

[22] The DNS Privacy Project. Initial Performance Measurements (Q1 2018). https://dnsprivacy.org/wiki/pages/ viewpage.action?pageId=14025132, 2018. Accessed: 2018-12-27.

[23] The DNS Privacy Project. Initial Performance Measurements (Q4 2018). https://dnsprivacy.org/wiki/pages/ viewpage.action?pageId=17629326, 2018. Accessed: 2018-12-27.

[24] Austin Hounsel, Kevin Borgolte, Paul Schmitt, Jordan Holland, and Nick Feamster. Analyzing the costs (and benefits) of DNS, DoT, and DoH for the modern web. CoRR, abs/1907.08089, 2019.

[25] Timm Bottger, Felix Cuadrado, Gianni Antichi, Eder Leao Fernandes, Gareth Tyson, Ignacio Castro, and Steve Uhlig. An empirical study of the cost of DNS-over-HTTPs. 2019.

[26] Brad Miller, Ling Huang, Anthony D Joseph, and J Doug Tygar. I know why you went to the clinic: Risks and realization of HTTPS traffic analysis. In Privacy Enhancing Technologies Symposium (PETS), pages 143163. Springer, 2014.

[27] Xiapu Luo, Peng Zhou, Edmond W. W. Chan, Wenke Lee, Rocky K. C. Chang, and Roberto Perdisci. HTTPOS: Sealing information leaks with browser-side obfuscation of encrypted flows. In Network \& Distributed System Security Symposium (NDSS). IEEE Computer Society, 2011.

[28] Milad Nasr, Amir Houmansadr, and Arya Mazumdar. Compressive traffic analysis: A new paradigm for scalable traffic analysis. In ACM SIGSAC Conference on Computer and Communications Security, CCS, 2017.

[29] DNS over Tor. https://developers.cloudflare.com/1.1.1.1/ fun-stuff/dns-over-tor/. Accessed: 2018-12-09.

[30] Tao Wang and Ian Goldberg. Improved Website Fingerprinting on Tor. In ACM Workshop on Privacy in the Electronic Society (WPES), pages 201-212. ACM, 2013.

[31] iodine. https://code.kryo.se/iodine/. Accessed: 2019-0513.

[32] Spamhaus. https://www.spamhaus.org/zen/. Accessed: 2019-05-13.

[33] Anonymous. Towards a comprehensive picture of the great firewall's dns censorship. In USENIX Security Symposium. USENIX Association, 2014.

[34] Paul Pearce, Ben Jones, Frank Li, Roya Ensafi, Nick Feamster, Nick Weaver, and Vern Paxson. Global measurement of dns manipulation. In USENIX Security Symposium. USENIX, page 22, 2017.

[35] Saikat Guha and Paul Francis. Identity trail: Covert surveillance using DNS. In Privacy Enhancing Technologies Symposium (PETS), 2007. 
[36] DNSSEC: DNS Security Extensions. https://www.dnssec. net/. Accessed: 2018-12-09.

[37] DNSCrypt. https://dnscrypt.info/. Accessed: 2018-12-09.

[38] G. Huston. DOH! DNS over HTTPS explained. https://blog.apnic.net/2018/10/12/ doh-dns-over-https-explained/. Accessed: 2018-1226.

[39] Kenji Baheux. Experimenting with sameprovider DNS-over-HTTPS upgrade. https: //blog.mozilla.org/futurereleases/2018/09/13/ dns-over-https-doh-testing-on-beta, 2019. accessed: 2019-09-13.

[40] T. Reddy, D. Wing, and P. Patil. Dns over datagram transport layer security (dtls). RFC 8094, RFC Editor, February 2017.

[41] Specification of DNS over Dedicated QUIC Connections. https://www.ietf.org/id/draft-huitema-quic-dnsoquic-05. txt. Accessed: 2018-12-09.

[42] Haya Shulman. Pretty bad privacy: Pitfalls of DNS encryption. In Proceedings of the 13th Workshop on Privacy in the Electronic Society. ACM, 2014.

[43] Dominik Herrmann, Christian Banse, and Hannes Federrath. Behavior-based tracking: Exploiting characteristic patterns in DNS traffic. Computers \& Security, 2013.

[44] Basileal Imana, Aleksandra Korolova, and John S. Heidemann. Enumerating Privacy Leaks in DNS Data Collected above the Recursive. 2017.

[45] DNS-OARC: Domain Name System Operations Analysis and Research Center. https://www.dns-oarc.net/tools/dsc. Accessed: 2018-11-26.

[46] DNS-STATS: ICANN's IMRS DNS Statistics. https:// www.dns.icann.org/imrs/stats. Accessed: 2018-12-06.

[47] Use DNS data to identify malware patient zero. https://docs.splunk.com/Documentation/ES/5.2.0/

Usecases/PatientZero. Accessed: 2018-12-06.

[48] DNS Analytics. https://constellix.com/dns/dns-analytics/. Accessed: 2018-12-06.

[49] Anonymous. The collateral damage of internet censorship by dns injection. SIGCOMM Comput. Commun. Rev., 42(3):21-27, 2012.

[50] Alerts about BGP hijacks, leaks, and outages. https:// bgpstream.com/. Accessed: 2019-05-13.

[51] Xiang Cai, Xin Cheng Zhang, Brijesh Joshi, and Rob Johnson. Touching from a distance: Website fingerprinting attacks and defenses. In ACM Conference on Computer and Communications Security (CCS), pages 605-616. ACM, 2012.

[52] Tao Wang, Xiang Cai, Rishab Nithyanand, Rob Johnson, and Ian Goldberg. Effective attacks and provable defenses for website fingerprinting. In USENIX Security Symposium, pages 143-157. USENIX Association, 2014.

[53] Vera Rimmer, Davy Preuveneers, Marc Juarez, Tom Van Goethem, and Wouter Joosen. Automated website fingerprinting through deep learning. In Network \& Distributed System Security Symposium (NDSS). Internet Society, 2018.

[54] Allison McDonald, Matthew Bernhard, Luke Valenta, Benjamin VanderSloot, Will Scott, Nick Sullivan, J Alex Halderman, and Roya Ensafi. 403 forbidden: A global view of cdn geoblocking. In Proceedings of the Internet Measurement Conference 2018, pages 218-230. ACM, 2018.
[55] Roberto Gonzalez, Claudio Soriente, and Nikolaos Laoutaris. User profiling in the time of https. In Proceedings of the 2016 Internet Measurement Conference, pages 373-379. ACM, 2016.

[56] Marc Liberatore and Brian Neil Levine. "Inferring the source of encrypted HTTP connections". In ACM Conference on Computer and Communications Security (CCS), pages 255-263. ACM, 2006.

[57] Kevin P. Dyer, Scott E. Coull, Thomas Ristenpart, and Thomas Shrimpton. Peek-a-Boo, I still see you: Why efficient traffic analysis countermeasures fail. In IEEE Symposium on Security and Privacy (S\&P), pages 332346. IEEE, 2012.

[58] Qixiang Sun, Daniel R Simon, Yi-Min Wang, Wilf Russel, Venkata N. Padmanabhan, and Lili Qiu. Statistical identification of encrypted web browsing traffic. In IEEE Symposium on Security and Privacy (S\&P), pages 19-30. IEEE, 2002.

[59] Andrew Hintz. Fingerprinting websites using traffic analysis. In Privacy Enhancing Technologies Symposium (PETS), pages 171-178. Springer, 2003.

[60] Dominik Herrmann, Rolf Wendolsky, and Hannes Federrath. Website fingerprinting: attacking popular privacy enhancing technologies with the multinomial NaïveBayes classifier. In ACM Workshop on Cloud Computing Security, pages 31-42. ACM, 2009.

[61] Andriy Panchenko, Lukas Niessen, Andreas Zinnen, and Thomas Engel. Website fingerprinting in onion routing based anonymization networks. In ACM Workshop on Privacy in the Electronic Society (WPES), pages 103114. ACM, 2011.

[62] Mario Almeida, Alessandro Finamore, Diego Perino, Narseo Vallina-Rodriguez, and Matteo Varvello. Dissecting dns stakeholders in mobile networks. In Proceedings of the 13th International Conference on emerging Networking EXperiments and Technologies, pages 28-34. ACM, 2017.

[63] David Plonka and Paul Barford. Context-aware clustering of dns query traffic. In Proceedings of the 8th ACM SIGCOMM conference on Internet measurement, pages 217-230. ACM, 2008.

[64] Rebekah Houser, Zhou Li, Chase Cotton, and Haining Wang. An investigation on information leakage of DNS over TLS. In Proceedings of the 15th International Conference on Emerging Networking Experiments And Technologies (CONEXT), pages 123-137, 2019.

[65] Rob Jansen, Marc Juarez, Rafael Galvez, Tariq Elahi, and Claudia Diaz. Inside job: Applying traffic analysis to measure tor from within. In Network \& Distributed System Security Symposium (NDSS). Internet Society, 2018.

[66] Trevor Hastie, Robert Tibshirani, and Jerome Friedman. The Elements of Statistical Learning. Springer Series in Statistics. Springer New York Inc., New York, NY, USA, 2001.

[67] Rebekah Overdorf, Marc Juarez, Gunes Acar, Rachel Greenstadt, and Claudia Diaz. How unique is your onion? an analysis of the fingerprintability of tor onion services. In ACM Conference on Computer and Communications Security (CCS), pages 2021-2036. ACM, 2017.

[68] Marc Juarez, Mohsen Imani, Mike Perry, Claudia Diaz, and Matthew Wright. Toward an efficient website finger- 
printing defense. In European Symposium on Research in Computer Security (ESORICS), pages 27-46. Springer, 2016.

[69] Ariel Stolerman, Rebekah Overdorf, Sadia Afroz, and Rachel Greenstadt. Breaking the closed-world assumption in stylometric authorship attribution. In IFIP Int. Conf. Digital Forensics, 2014.

[70] Muhammad Ahmad Bashir and Christo Wilson. Diffusion of user tracking data in the online advertising ecosystem. 2018.

[71] Marc Juarez, Sadia Afroz, Gunes Acar, Claudia Diaz, and Rachel Greenstadt. A critical evaluation of website fingerprinting attacks. In ACM Conference on Computer and Communications Security (CCS), pages 263-274. ACM, 2014

[72] John S Otto, Mario A Sánchez, John P Rula, and Fabián E Bustamante. Content delivery and the natural evolution of DNS: remote dns trends, performance issues and alternative solutions. In Proceedings of the 2012 Internet Measurement Conference, pages 523-536. ACM, 2012.

[73] John P Rula and Fabian E Bustamante. Behind the curtain: Cellular dns and content replica selection. In Proceedings of the 2014 Conference on Internet Measurement Conference, pages 59-72. ACM, 2014.

[74] Platon Kotzias, Abbas Razaghpanah, Johanna Amann, Kenneth G Paterson, Narseo Vallina-Rodriguez, and Juan Caballero. Coming of age: A longitudinal study of tls deployment. In Proceedings of the Internet Measurement Conference, pages 415-428. ACM, 2018.

[75] J. Damas, M. Graff, and P. Vixie. Extension mechanisms for dns (edns(0)). RFC 6891, RFC Editor, April 2013.

[76] Padding Policies for Extension Mechanisms for DNS (EDNS(0)). https://tools.ietf.org/html/rfc8467. Accessed: 2019-05-10.

[77] Michael Carl Tschantz, Sadia Afroz, Anonymous, and Vern Paxson. Sok: Towards grounding censorship circumvention in empiricism. In IEEE Symposium on Security and Privacy (S\&P), pages 914-933. IEEE, 2016.

[78] Sheharbano Khattak, Tariq Elahi, Laurent Simon, Colleen M Swanson, Steven J Murdoch, and Ian Goldberg. SoK: Making sense of censorship resistance systems. Privacy Enhancing Technologies Symposium (PETS), 2016(4):37-61, 2016.

[79] URL testing lists intended for discovering website censorship. https://github.com/citizenlab/test-lists. Accessed: 2019-09-11.

[80] Paul Schmitt, Anne Edmundson, Allison Mankin, and Nick Feamster. Oblivious dns: practical privacy for dns queries. Proceedings on Privacy Enhancing Technologies, 2019(2):228-244, 2019.

[81] Davis Yoshida and Jordan Boyd-Graber. Using confusion graphs to understand classifier error. In Proceedings of the Workshop on Human-Computer Question Answering, pages 48-52, 2016.

\section{APPENDIX}

\section{A. Datasets}

Table IX provides an expanded version of the dataset overview.

\section{B. Performance metrics.}

In this section, we provide an overview of our classifer evaluation metrics. We use standard metrics to evaluate the performance of our classifier: Precision, Recall and F1-Score. We compute these metrics per class, where each class represents a webpage. We compute these metrics on a class as if it was a "one vs. all" binary classification: we call "positives" the samples that belong to that class and "negatives" the samples that belong to the rest of classes. Precision is the ratio of true positives to the total number of samples that were classified as positive (true positives and false positives). Recall is the ratio of true positives to the total number of positives (true positives and false negatives). The F1-score is the harmonic mean of precision and recall.

\section{Estimation of Probabilities}

In this section, we explain how we estimated the probabilities for the entropy analysis in Section VII.

We define the anonymity set of a trace $s$ as a multiset:

$$
A(s):=\left\{w^{\mathrm{m}_{s}(w)}\right\}
$$

where $\mathrm{m}_{s}(w)$ is the multiplicity of a website $w$ in $A(s)$. The multiplicity is a function defined as the number of times that trace $s$ occurrs in $w$.

The probability $\operatorname{Pr}\left[W=w \mid S_{l}=o\right]$ can be worked out using Bayes. For instance, for website $w$,

$$
\operatorname{Pr}\left[W=w \mid S_{l}=o\right]=\frac{\operatorname{Pr}[W=w] \operatorname{Pr}\left[S_{l}=o \mid W=w\right]}{\sum_{i=1}^{m} \operatorname{Pr}\left[W=w_{i}\right] \operatorname{Pr}\left[S_{l}=o \mid W=w_{i}\right]}
$$

We assume the distribution of priors is uniform, i.e., the probability of observing a website is the same for all websites: $\operatorname{Pr}\left[w_{i}\right]=\operatorname{Pr}\left[w_{j}\right] \quad \forall i, j$.

We acknowledge that this is an unrealisitc assumption but we provide the mathematical model to incorporate the priors in case future work has the data to estimate them.

Assuming uniform priors simplifies the Bayes rule formula since we can factor out $\operatorname{Pr}\left[W=w_{i}\right]$ in Equation 1

Regarding the likelihoods of observing the traces given a website, we can use the traffic trace samples in our dataset as observations to estimate them:

$$
\operatorname{Pr}\left[S_{l}=o \mid W=w_{i}\right] \approx \frac{\mathrm{m}_{s}\left(w_{i}\right)}{k_{i}} .
$$

Since we have a large number of samples for all the sites, we can fix the same sample size for all sites: $k_{i}=k_{j} \quad \forall i, j$. A fixed sample size allows us to factor out $k_{i}$ in our likelihood estimates and, thus, the posterior can be estimated as:

$$
\operatorname{Pr}\left[W=w \mid S_{l}=o\right] \approx \frac{\mathrm{m}_{s}(w)}{\sum_{i=1}^{m} \mathrm{~m}_{s}\left(w_{i}\right)}=\frac{\mathrm{m}_{s}(w)}{|A(s)|}
$$

That is the multiplicity of website $w$ divided by the size of the $s$ 's anonymity set, which can be computed efficiently for all $w$ and $s$ using vectorial operations. 
Table IX: Overview of datasets (expanded).

\begin{tabular}{|c|c|c|c|c|c|c|c|}
\hline Name & Identifier & Location & Resolver & Client & Platform & \# webpages & \# samples \\
\hline Desktop (Location 1) & LOC1 & Lausanne & Cloudflare & Cloudflare & Desktop & 1,500 & 200 \\
\hline Desktop (Location 2) & LOC2 & Leuven & Cloudflare & Cloudflare & Desktop & 1,500 & 60 \\
\hline Desktop (Location 3) & LOC3 & Singapore & Cloudflare & Cloudflare & Desktop (AWS) & 1,500 & 60 \\
\hline Raspberry $\mathrm{Pi}$ & RPI & Lausanne & Cloudflare & Cloudflare & Raspberry Pi & 700 & 60 \\
\hline Firefox with Google resolver & GOOGLE & Leuven & Google & Firefox & Desktop & 700 & 60 \\
\hline Firefox with Cloudflare resolver & CLOUD & Leuven & Cloudflare & Firefox & Desktop & 700 & 60 \\
\hline Firefox with Cloudflare client & CL-FF & Leuven & Cloudflare & Cloudflare & Desktop & 700 & 60 \\
\hline Open World & OW & Lausanne & Cloudflare & Cloudflare & Desktop & 5,000 & 3 \\
\hline DoH and web traffic & WEB & Leuven & Cloudflare & Cloudflare & Desktop & 700 & 60 \\
\hline DNS over Tor & TOR & Lausanne & Cloudflare & Cloudflare & Desktop & 700 & 60 \\
\hline Cloudflare's EDNS0 padding implementation & EDNS0-128 & Lausanne & Cloudflare & Cloudflare & Desktop & 700 & 60 \\
\hline Recommended EDNS0 padding & EDNS0-468 & Lausanne & Cloudflare & Cloudflare & Desktop & 700 & 60 \\
\hline EDNS0 padding with ad-blocker & EDNS0-128-adblock & Lausanne & Cloudflare & Cloudflare & Desktop & 700 & 60 \\
\hline DoT with Stubby client & DOT & Lausanne & Cloudflare & Stubby & Desktop & 700 & 60 \\
\hline
\end{tabular}

\section{Extra results on attack robustness}

In this section, we provide additional information on our experiment measuring the influence of end user's platform (Section V-C3). Figure 12 shows the difference in TLS record sizes for both platforms - the sizes follow a similar distribution, with a shift. Table $\mathrm{X}$ shows the improvement in performance we obtain when removing this shift.

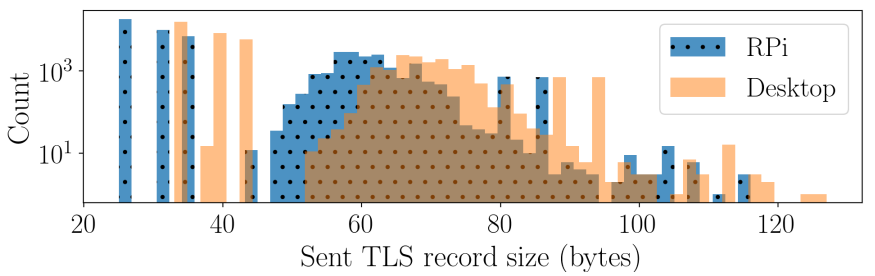

Figure 12: Distribution of user's sent TLS record sizes in platform experiment.

Table X: Improvement in cross platform performance when removing the shift (standard deviation less than 1\%).

\begin{tabular}{ccccc}
\hline Train & Test & Precision & Recall & F-score \\
\hline DESKTOP & RPI & 0.630 & 0.654 & 0.614 \\
RPI & DESKTOP & 0.552 & 0.574 & 0.535 \\
\hline
\end{tabular}

\section{E. Survivors and Easy Preys}

In this section, we show results from our analysis of survivors and easy preys, as discussed in Section V-C. We show the top-10 webpages with highest-mean and lowest-variance (Table XI), lowest-mean and lowest-variance (Table XII), and highest-variance F1-score (Table XIII).

\section{F. Confusion Graphs}

We have used confusion graphs to understand the errors of the classifier. Confusion graphs are the graph representation of confusion matrices. They allow to easily visualize large confusion matrices by representing misclassifications as directed graphs. Confusion graphs have been used in website fingerprinting [67] and other classification tasks to understand classifier error [81]. The graphs for our classifier can be found in the extended version of our paper at https://github.com/spring-epfl/doh_traffic_analysis/blob/ master/paper/doh_traffic_analysis_ndss2020.pdf.
Table XI: Top-10 with highest-mean and lowest-variance F1Score

\begin{tabular}{rrrl}
\hline Alexa Rank & Mean F1-Score & Stdev F1-Score & Domain name \\
\hline 777 & 0.95 & 0.08 & militaryfamilygiftmarket.com \\
985 & 0.95 & 0.08 & myffpc.com \\
874 & 0.95 & 0.08 & montrealhealthygirl.com \\
712 & 0.95 & 0.08 & mersea.restaurant \\
1496 & 0.95 & 0.08 & samantha-wilson.com \\
1325 & 0.95 & 0.08 & nadskofija-ljubljana.si \\
736 & 0.95 & 0.08 & michaelnewnham.com \\
852 & 0.95 & 0.08 & mollysatthemarket.net \\
758 & 0.95 & 0.08 & midwestdiesel.com \\
1469 & 0.95 & 0.08 & reclaimedbricktiles.blogspot.si \\
\hline
\end{tabular}

Table XII: Top-10 sites with lowest-mean and lowest-variance F1-Score

\begin{tabular}{rrrl}
\hline Alexa Rank & Mean F1-Score & Stdev F1-Score & Domain name \\
\hline 822 & 0.11 & 0.10 & mjtraders.com \\
1464 & 0.11 & 0.08 & ravenfamily.org \\
853 & 0.14 & 0.09 & moloneyhousedoolin.ie \\
978 & 0.14 & 0.17 & mydeliverydoctor.com \\
999 & 0.17 & 0.10 & myofascialrelease.com \\
826 & 0.17 & 0.11 & mm-bbs.org \\
1128 & 0.17 & 0.10 & inetgiant.com \\
889 & 0.18 & 0.14 & motorize.com \\
791 & 0.18 & 0.15 & mindshatter.com \\
1193 & 0.20 & 0.14 & knjiznica-velenje.si \\
\hline
\end{tabular}

Table XIII: Top-10 sites with highest-variance F1-Score

\begin{tabular}{rrrl}
\hline Alexa Rank & Mean F1-Score & Stdev F1-Score & Domain name \\
\hline 1136 & 0.43 & 0.53 & intothemysticseasons.tumblr.com \\
782 & 0.43 & 0.53 & milliesdiner.com \\
766 & 0.43 & 0.53 & mikaelson-imagines.tumblr.com \\
1151 & 0.43 & 0.53 & japanese-porn-guidecom.tumblr.com \\
891 & 0.42 & 0.52 & motorstylegarage.tumblr.com \\
909 & 0.42 & 0.52 & mr-kyles-sluts.tumblr.com \\
918 & 0.44 & 0.52 & mrsnatasharomanov.tumblr.com \\
1267 & 0.52 & 0.49 & meander-the-world.com \\
238 & 0.48 & 0.49 & caijing.com.cn \\
186 & 0.48 & 0.48 & etsy.com \\
\hline
\end{tabular}

